\title{
Predicting Hydrophobic Solvation by Molecular Simulation: 2. New United-atom Model for Alkanes, Alkenes and Alkynes
}

\author{
Miguel Jorge* \\ Department of Chemical and Process Engineering, University of Strathclyde, 75 Montrose \\ Street, Glasgow G1 1XJ, United Kingdom \\ Email-miguel.jorge@strath.ac.uk
}

\begin{abstract}
Existing united-atom models for non-polar hydrocarbons lead to systematic deviations in predicted solvation free energies in hydrophobic solvents. In this paper, an improved set of parameters is proposed for alkane molecules that corrects this systematic deviation and accurately predicts solvation free energies in hydrophobic media, while simultaneously providing a very good description of pure liquid densities. The model is then extended to alkenes and alkynes, again yielding very accurate predictions of solvation free energies and densities for these classes of compounds. For alkynes in particular, this work represents the first attempt at a systematic parameterization using the united-atom approach. Averaging over all 95 solute/solvent pairs tested, the mean signed deviation from experimental data is very close to zero, indicating no systematic error in the predictions. The fact that predictions are robust even for relatively large molecules suggests that the new model may be applicable to solvation of non-polar macromolecules without accumulation of errors. The root mean squared deviation of the simulations is only $0.6 \mathrm{~kJ} / \mathrm{mol}$, which is lower than the estimated uncertainty in the experimental measurements. This excellent performance constitutes a solid basis upon which a more general model can be parameterized to describe solvation in both polar and non-polar environments.
\end{abstract}

Keywords: Solubility; Molecular Simulation; hydrocarbons; non-polar; free energy 


\section{1 - Introduction}

Predicting solvation in hydrophobic environments is relevant for a wide range of processes, from industrial separations to protein-ligand binding [1-4]. However, it has been largely overlooked in previous molecular simulation studies, which have primarily focused on aqueous solvation (or hydration) processes [5-7]. Moreover, most interaction potential models, or force-fields, suitable for use in solution have been parameterized against bulk liquid properties. For example, the widely used OPLS model was parameterized to match pure liquid densities and enthalpies of vaporization [8]. A notable exception to this trend is a recent version of the GROMOS force-field [9], where experimental solvation free energies were used as target properties in the parameterization procedure. Interestingly, the authors developed two alternative version of the model, one optimized for pure liquid properties (version 53A5) and another for solvation free energy calculations (version 53A6). However, the parameters for alkanes, the archetypal hydrophobic molecules, were taken directly from a previous parameter set [10], where pure liquid densities and enthalpies of vaporization were again used as target properties while hydration free energies were only used for subsequent validation. With the exception of a recent study by Szklarczyk et al. [14] reporting excess free energies, which are related to the self-solvation free energies, for a few alkane molecules using GROMOS 45A3 parameters, the quality of those alkane parameters has not yet been fully tested in the context of hydrophobic solvation free energies.

A particularly successful class of models for alkanes are united atom (UA) models. In this approach, $\mathrm{CH}_{\mathrm{x}}$ groups are taken as a single interaction site - i.e., hydrogen atoms are lumped together into the adjacent carbon atom. Because alkane hydrogen atoms are not modelled explicitly, each interaction site is taken to be electronically neutral, so that electrostatic interactions can be neglected altogether. The UA approximation not only speeds up the calculations significantly due to the reduced number of interaction sites and neglect of electrostatics, but also, crucially, simplifies the parameterization procedure by reducing the number of free fitting variables in the model. Both of these advantages are of great importance for the present study, as solvation free energy calculations are quite computationally demanding and normally require a separate expensive calculation to account for the electrostatic component. The UA approach has been shown to be a reasonable approximation for non-polar hydrocarbons, leading to generally good predictions of static fluid properties $[8,10]$ and phase equilibrium [11-14]. However, they tend to perform worse than their all-atom counterparts in predictions of dynamic properties (e.g., diffusion and viscosity) [15] because the coarse-graining of the interaction sites leads to less accurate dynamics. Moreover, the complete neglect of electrostatics and polarization means that they are unable to predict dielectric properties, although all-atom fixed-charge models do not appear to perform much better in this respect [16].

The previous paper of this series [17] compared the performance of three popular UA alkane models, OPLS-UA [8], GROMOS [10] and TraPPE [11-14], for predicting 
hydrophobic solvation, i.e., solvation free energies of alkane solutes in alkane solvents. It was found that all three force-fields showed systematic deviations from experimental data [18, 19], with OPLS-UA and GROMOS overestimating the magnitude of solvation (by $15 \%$ and 13\%, respectively), and TraPPE slightly underestimating it (by 6\%) [17]. This performance was rationalized on the basis of the parameterization strategy and target experimental properties used by each model. The fact that the deviations are systematic implies that they will accumulate for macromolecules with large hydrophobic domains, such as polymers and proteins, with potentially profound impact in their solvation behavior. It also suggests that the models can be improved by relatively small changes in the interaction parameters. In this paper, such a possibility is explored, leading to an optimized set of alkane UA parameters for prediction of hydrophobic solvation free energies. The starting point is the TraPPE model because it performed best [17], despite the fact that solvation free energies were never used in its parameterization or validation. Slightly changing the Lennard-Jones (LJ) interaction parameters leads to excellent agreement with experiment for over 50 solute-solvent pairs that include linear, branched and cyclic alkanes. The representation of cyclic alkanes was also simplified, using a single set of parameters for this class of molecule (as opposed to three different parameter sets in the original TraPPE model). Finally, the approach was extended to unsaturated hydrocarbons, namely alkenes and alkynes, thus completing the new force-field for aliphatic hydrocarbons. This improved model forms a strong basis for the development of a general force-field that is optimized for predicting solvation free energies of compounds with a wide range of polarities.

\section{2 - Computational Methods}

Details of the computational procedure were given in the first paper of this series [17], as well as in previous publications [20-25]. Briefly, solvation free energies were calculated by the thermodynamic integration (TI) method [26] based on a series of molecular dynamics (MD) simulations carried out using the GROMACS software [27]. TI relies on applying a coupling parameter, $\lambda$, to the solute-solvent part of the Hamiltonian, which is then changed gradually between full interactions (corresponding to $\lambda=0$ ) and no interactions $(\lambda=1)$. Essentially, the solute is made to gradually "disappear" from the solution using the coupling parameter. A series of independent MD simulations were carried out for different values of $\lambda$ and the gradient of the Hamiltonian with respect to $\lambda$ was averaged over a large number of equilibrated configurations. The solvation free energy $\left(\Delta G_{\text {sol }}\right)$ was then calculated by numerically integrating the Hamiltonian gradient over $\lambda$ [25]. Note that because the systems studied in this paper involve non-polar alkanes described at the UA level, only the LennardJones contribution to the solvation free energy needs to be considered, and no separate calculation of the electrostatic component is needed.

In this work, a total of $15 \lambda$ points were used. For each of these points, 50 independent 200 ps simulations were carried out starting from different initial configurations. This 
allowed the calculations to be run most effectively on the volunteer computing platform for the Iberian Peninsula, IBERCIVIS [28]. In the previous paper [17], it was demonstrated that this approach led to appropriately converged results. Each MD simulation was performed in the isothermal-isobaric ensemble, thus yielding the Gibbs free energy of solvation. Temperature was kept fixed at $298 \mathrm{~K}$ using a Langevin thermostat [29] and pressure was fixed at 1 bar using a Parinello-Rahman barostat [30]. The equations of motion were integrated using the leapfrog algorithm [31] with a time step of $2 \mathrm{fs}$. The only exception to this protocol was for simulations involving alkynes, for which the Langevin dynamics integrator was causing unphysical distortions of the $180^{\circ}$ angle involving the triple bond (see Table S1). These were thus run using the conventional MD integrator and a Nose-Hoover thermostat, which eliminated the problem. A switched cut-off between 1.0 and $1.1 \mathrm{~nm}$ was used for dispersion interactions and long-range dispersion corrections were applied to both energy and pressure. Use of these long-range corrections ensures that the free energy results are independent of cutoff radius, provided it is at least $0.9 \mathrm{~nm}[17,32]$.

Table 1 - Lennard-Jones parameters for the new united-atom force-field for aliphatic hydrocarbons proposed in this paper (bonded parameters for alkanes and alkenes are the same as in the original TraPPE model, while those for alkynes were taken from OPLS-AA [33] - see also Table S1). All sites are electronically neutral by construction.

\begin{tabular}{l|l|c|c} 
Molecule type & Site & $\sigma(\mathrm{nm})$ & $\varepsilon(\mathrm{kJ} / \mathrm{mol})$ \\
\hline \multirow{2}{*}{ Alkanes $\left(s p^{3}\right)$} & $\mathrm{CH}_{4}$ & 0.371 & 1.200 \\
\cline { 2 - 4 } & $\mathrm{CH}_{3}$ & 0.379 & 0.833 \\
\cline { 2 - 4 } & $\mathrm{CH}_{2}$ (linear and branched) & 0.399 & 0.392 \\
& $\mathrm{CH}_{2}$ (cyclic) & 0.392 & 0.450 \\
\cline { 2 - 4 } & $\mathrm{CH}$ & 0.473 & 0.0850 \\
\cline { 2 - 4 } & $\mathrm{C}$ & 0.646 & 0.00426 \\
\hline Alkenes $\left(s p^{2}\right)$ & $\mathrm{CH}_{2}$ & $0.3675^{*}$ & $0.7067^{*}$ \\
\cline { 2 - 4 } & $\mathrm{CH}$ & $0.373^{*}$ & $0.39076^{*}$ \\
\cline { 2 - 4 } & $\mathrm{CH}$ (conjugated) & $0.371^{*}$ & $0.43233^{*}$ \\
\cline { 2 - 4 } & $\mathrm{C}$ & $0.385^{*}$ & $0.16628^{*}$ \\
\hline Alkynes $(s p)$ & $\mathrm{CH}$ & 0.3315 & 0.628 \\
\cline { 2 - 4 } & $\mathrm{C}$ & 0.380
\end{tabular}

*Parameters were kept identical to the original TraPPE model.

As explained previously, the starting point for the improved model is the TraPPE force-field. Bonded parameters were kept the same as in the original TraPPE model, as they lead to a satisfactory description of alkane conformations in the liquid state [11-14] and their impact on solvation free energies is likely to be minor. For the alkynes, the bonded parameters from OPLS-AA [33] were used (Table S1), as these were not available in TraPPE. Attention was thus focused on tuning the LJ parameters to improve solvation free energy predictions. The database of Katritzky et al. $[18,19]$ was used for experimental solvation free 
energy data, but additional data from Wolfenden and co-workers [34] and from the Minnesota Solvation Database [35, 36] was used for model validation where explicitly specified. For some fluids, bulk liquid densities $(\rho)$ were calculated by sampling over equilibrated pure liquid simulations in the $\mathrm{NpT}$ ensemble, and enthalpies of vaporization $\left(\Delta H_{\text {vap }}\right)$ were computed using the following equation:

$$
\Delta H_{v a p}=U_{g a s}-U_{l i q}+R T
$$

In equation (1), $U_{\text {liq }}$ is the molar potential energy in the liquid phase, obtained from averaging over a pure liquid simulation, $U_{\text {gas }}$ is the potential energy in the vapor phase, calculated from simulations of a single molecule in vacuum with no periodic boundary conditions, $R$ is the ideal gas constant and $T$ is the temperature. Adequate conformational sampling in both the liquid and gas phases was confirmed by monitoring dihedral angle distributions. Experimental densities were taken from Weast and Astle [37], while experimental vaporization enthalpies and associated uncertainties were taken from NIST [38]. The optimized set of parameters for all types of aliphatic hydrocarbons is provided in Table 1 of this paper (see also Supplementary Material). The parameterization approach used for each class of molecules is explained in detail in the results section.

\section{3 - Results and discussion}

\section{1 - Cyclic Alkanes}

As discussed in the first paper of this series [17], the choice of parameterization strategy can have a profound impact on the performance of the force-field, particularly when it is used beyond the original set of target molecules and/or properties. For instance, the performance of OPLS-UA deteriorates significantly for larger alkane molecules largely because it employs the same set of parameters for $\mathrm{CH}_{2}$ groups in linear, branched and cyclic alkanes. This was later shown to be an unfortunate choice, as the additional excluded volume within the ring needs to be compensated by the use of specific interaction parameters for cyclic molecules $[13,39]$. Because $\mathrm{CH}_{2}$ parameters in OPLS-UA were first benchmarked against properties of pure cyclopentane and were then carried over to linear alkanes [8], the parameters for $\mathrm{CH}_{3}$ groups needed to compensate for the overestimated attractiveness of $\mathrm{CH}_{2}$ groups. This was achieved for small molecules at the cost of increased complexity (different $\mathrm{CH}_{3}$ parameters for different classes of alkanes), but led to increased inaccuracy for large alkanes.

Conversely, the most recent version of TraPPE [14] adopts different parameters for $\mathrm{CH}_{2}$ groups in cyclic alkanes of different sizes (more specifically, 3 different parameter sets were proposed, for cyclopentane, for cyclohexane and for molecules larger than cycloheptane, not including the latter). Our comparison of existing force-fields against experimental data for solvation of cyclic alkanes (see Figure 11 of the previous paper [17]) shows no evidence that TraPPE qualitatively outperforms GROMOS and OPLS-UA for this 
class of molecules, despite the added complexity. I believe the optimal balance between complexity and accuracy lies in using two different sets of parameters, one for cyclic alkanes and another for linear and branched alkanes (which, incidentally, is the approach used by the GROMOS force-field). As such, it was decided to explore the possibility of using a single set of parameters for $\mathrm{CH}_{2}$ groups in cyclic alkanes, calibrated against properties of pure cyclohexane. This is the ideal test case, as the system contains only the type of site that one wishes to parameterize. Also, cyclohexane is a widely used solvent, so this system assumes particular relevance for future applications of the model. As target experimental properties, the density of the liquid [37], the enthalpy of vaporization [38] and the self-solvation free energy (i.e., for cyclohexane solute dissolved in cyclohexane solvent) $[18,19]$ were chosen.

Analyzing the parameters for cyclic $\mathrm{CH}_{2}$ groups in the 3 force-fields considered earlier (see Table 1 of the previous paper [17]), it can be seen that they are spread over a relatively narrow range of values around $\sigma \approx 0.39 \mathrm{~nm}$ and $\varepsilon \approx 0.46 \mathrm{~kJ} / \mathrm{mol}$. Therefore, the sensitivity of the three different target properties to $\sigma$ and $\varepsilon$ was probed over a narrow window roughly centered on those values. Admittedly, this is a rather computationally expensive way to parameterize a model. However, the results provide a better understanding of how each property changes with each of the LJ parameters. Such an understanding will facilitate further parameterization efforts.

Figure 1 shows that the liquid density decreases linearly with $\sigma$ and increases with $\varepsilon$ in a non-linear fashion within this range of values. Qualitatively speaking, this is expected, as an increase in $\sigma$ increases the excluded volume of each molecule, thus decreasing the density, while increasing $\varepsilon$ increases the cohesive energy of the fluid, making it denser. Figure 2 shows analogous results for the enthalpy of vaporization. Here we see a practically linear increase in $\Delta H_{\text {vap }}$ with both $\sigma$ and $\varepsilon$ in this range of values. Both of these trends are likely to be caused by an increase in the cohesive energy of the liquid as both $\sigma$ and $\varepsilon$ increase (the increase in excluded volume due to increase in $\sigma$ seems to play a negligible role in $\Delta H_{\text {vap}}$ ).

For the self-solvation free energy (Figure 3), a similar trend as for $\Delta H_{\text {vap }}$ is observed, except that the sign of the gradients is reversed (recall that the vaporization and solvation processes take place in opposite directions between the gas and liquid/solution phases). The trend with increasing $\varepsilon$ is once again caused by the stronger solute-solvent interactions, which favors solvation (i.e., $\Delta G$ is more negative). The trend of more favorable solvation with increasing $\sigma$, however, is not as trivial. It can be rationalized by considering two competing effects at play: an increase in solute-solvent interactions which is manifested in the increase of $\Delta H_{\text {vap }}$ with $\sigma$, and an increase in the excluded volume of both solvent and solute molecules, which is manifested in the decrease of density with $\sigma$. These effects influence $\Delta G$ in opposite ways, since an increase in the volume of the solute will tend to increase the cavity formation cost, thus making $\Delta G$ more positive. However, it appears that within this range of 
values, the influence of the solute-solvent attraction dominates and the excluded volume effect is rather minor.
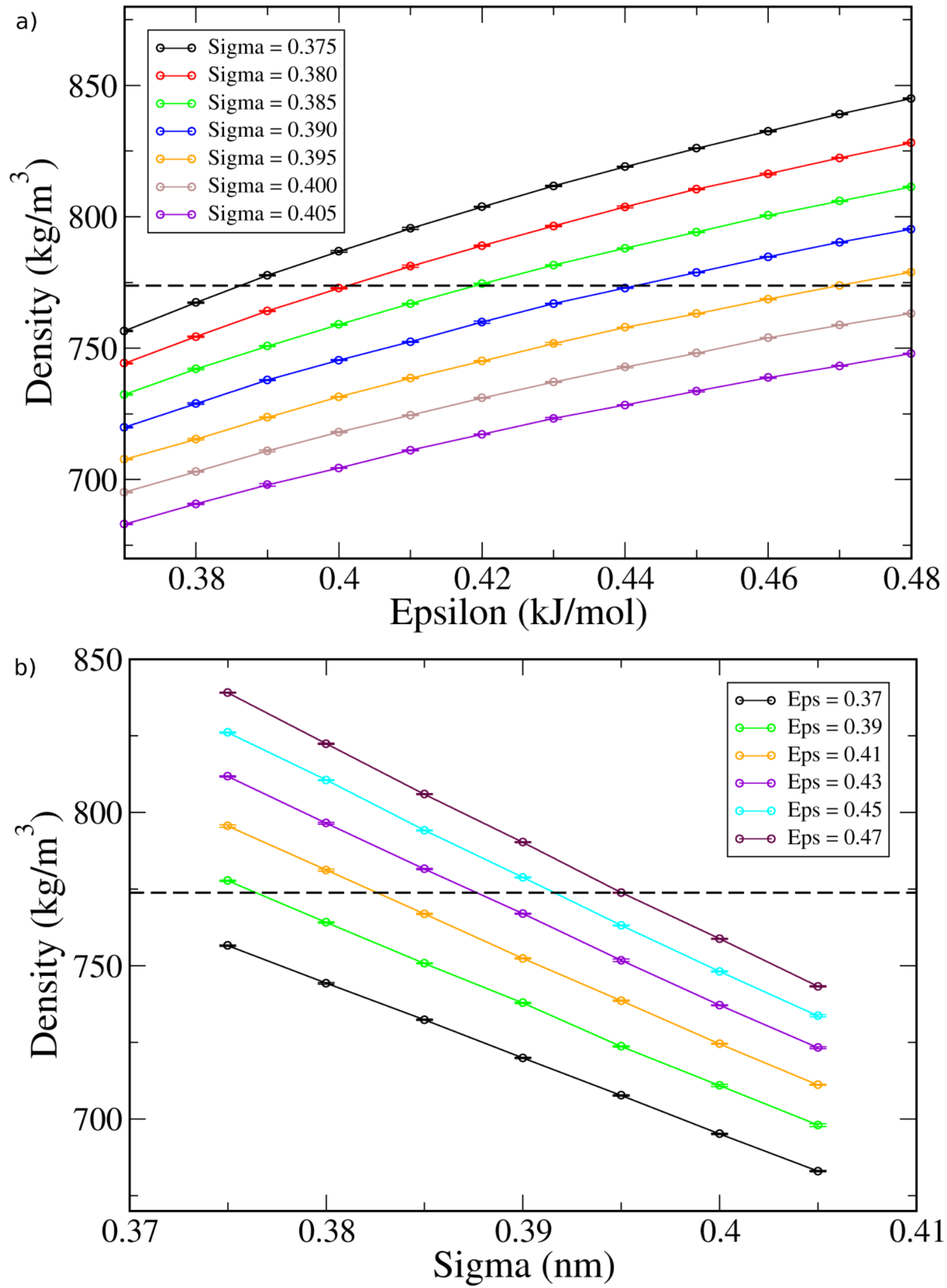

Figure 1 - Density of pure cyclohexane solvent for different values of the Lennard-Jones parameters for the cyclic $\mathrm{CH}_{2}$ group: a) epsilon; b) sigma. The horizontal thick dashed line shows the experimental value [37] (experimental uncertainty is assumed negligible). 

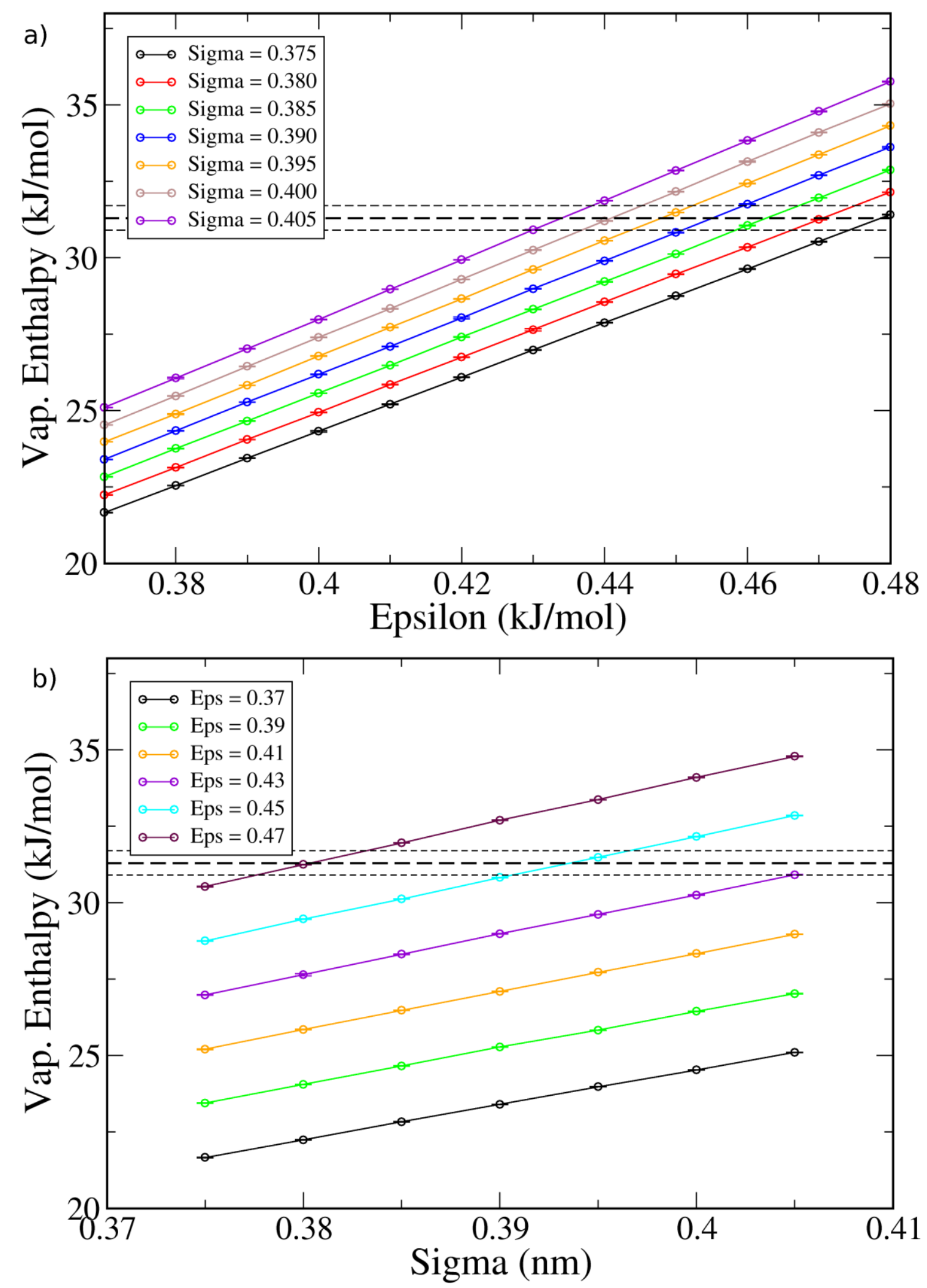

Figure 2 - Enthalpy of vaporization of cyclohexane for different values of the Lennard-Jones parameters for the cyclic $\mathrm{CH}_{2}$ group: a) epsilon; b) sigma. The horizontal thick dashed line shows the experimental value, while the thin dashed lines represent upper and lower bounds based on the reported uncertainty in the experimental measurements [38]. 

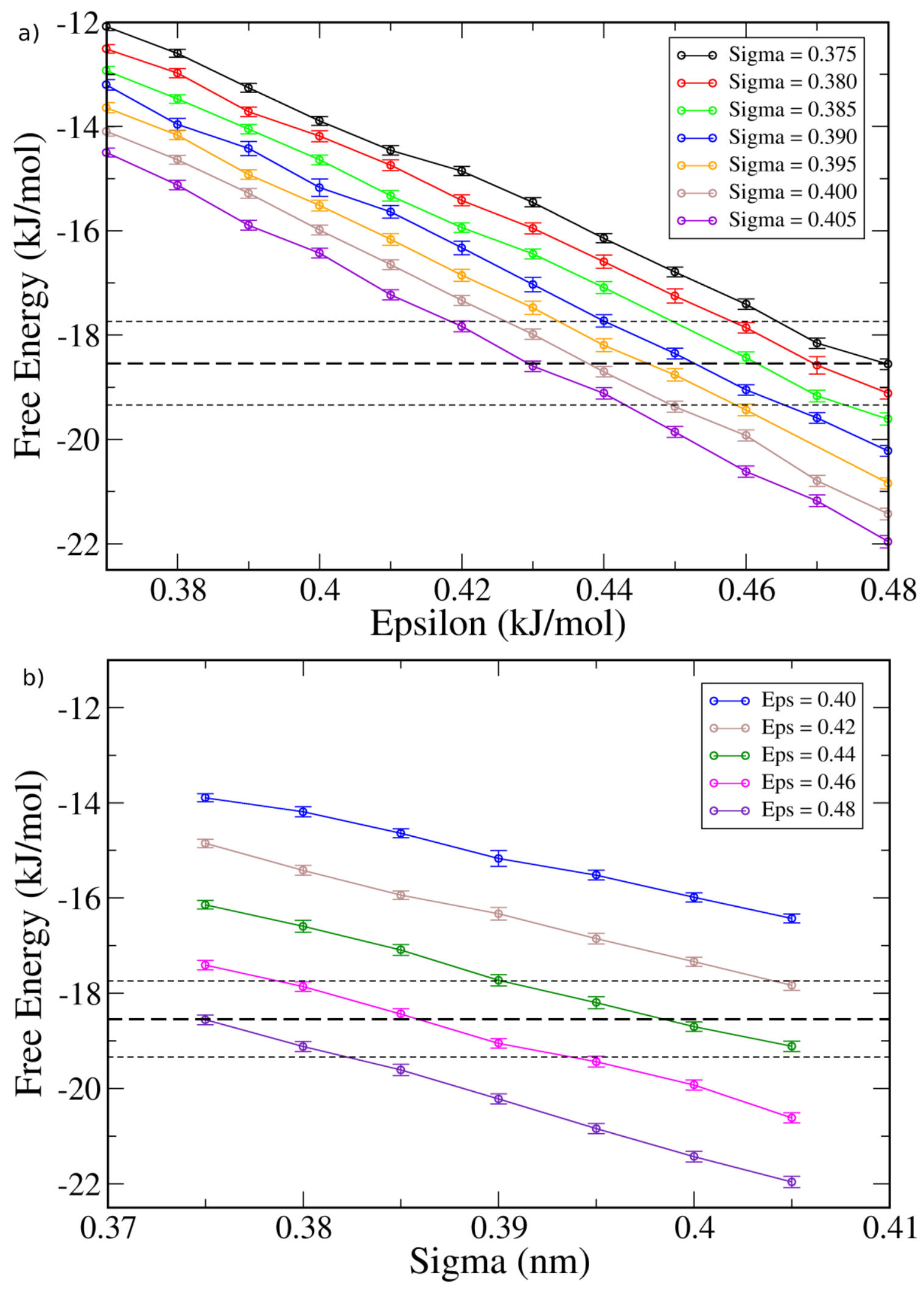

Figure 3 - Solvation free energy of cyclohexane solute in cylcohexane solvent (self-solvation) for different values of the Lennard-Jones parameters for the cyclic $\mathrm{CH}_{2}$ group: a) epsilon; b) sigma. The horizontal thick dashed line shows the experimental value [18, 19], while the thin dashed lines represent upper and lower bounds based on the estimated uncertainty in experimental measurements [40]. 
Also shown in Figures 1-3 are the experimental values for each property, with corresponding uncertainties (for density, this is assumed to be negligible). It is clear that for a given property there exists a potentially infinite set of $(\sigma, \varepsilon)$ pairs that can match the experimental value. As expected, one needs at least two experimental properties to unambiguously determine the optimal values of the two parameters. Figure 4 shows trajectories in $(\sigma, \varepsilon)$ space that correspond to a perfect match between simulation and each of the three experimental properties. As one can see, the curves for $\Delta H_{\text {vap }}$ and $\Delta G$ are nearly parallel, which is a consequence of the similar trends shown in Figures 2 and 3. The density, however, shows a completely different trajectory, given that it changes with $\sigma$ and $\varepsilon$ in different ways than $\Delta H_{\text {vap }}$ and $\Delta G$. This suggests that density is a good property to use in force-field calibration in combination with either $\Delta H_{\text {vap }}$ or $\Delta G$. It is perhaps no coincidence that most early efforts to parameterize force-fields for liquids (e.g., OPLS and early versions of GROMOS) used precisely the density and vaporization enthalpy of the pure liquids.

Another important observation from Figure 4 is that parameter pairs that provide a good match to $\Delta H_{\text {vap }}$ also do a very good job at predicting $\Delta G$, at least for the range tested. This suggests that $\Delta H_{\text {vap }}$ might be used as a cheaper alternative to $\Delta G$ for force field parameterization, although further work with other types of liquid (including polar compounds) is needed to fully ascertain this. In any case, it is possible to find a unique pair of parameters that matches all three properties within the level of experimental uncertainty. The final LJ parameters for $\mathrm{CH}_{2}$ groups in cyclic alkanes are $\sigma=0.392 \mathrm{~nm}$ and $\varepsilon=0.450 \mathrm{~kJ} / \mathrm{mol}$ (Table 1). It will be shown later that the same parameters also provide a good description of solvation free energies of different cyclic alkanes in n-hexadecane.

Perhaps not surprisingly, the optimal set of parameters is quite similar to those of the TraPPE model for $\mathrm{CH}_{2}$ groups in cyclohexane, $\sigma=0.391 \mathrm{~nm}$ and $\varepsilon=0.4365 \mathrm{~kJ} / \mathrm{mol}$, and not very different from the corresponding parameters in GROMOS and OPLS-UA (see Table 1 of the first paper [17]). The slight underestimation of solvation in the original TraPPE and overestimation in the other two force-fields is corrected mainly by using an intermediate value of $\varepsilon$. The new model also provides closer agreement with experimental density than any of the previous force-fields. The self-solvation free energy is related to the vapor pressure of the pure component [41], so the new model is expected to also provide an accurate description of the vapor pressure of alkanes. Indeed, the underestimation of solvation by TraPPE can be traced back to the underestimation of the vapor pressure in that model [11], as discussed in our first paper [17]. It is important to recall that in the original TraPPE model, the authors chose to sacrifice agreement with the vapor pressure in favor of a closer match to experimental critical properties. As a consequence, one should expect the new model to lead to slightly worse predictions of critical properties than the original TraPPE. A detailed assessment of the performance of the new model in vapor-liquid equilibrium properties is beyond the scope of this paper. 


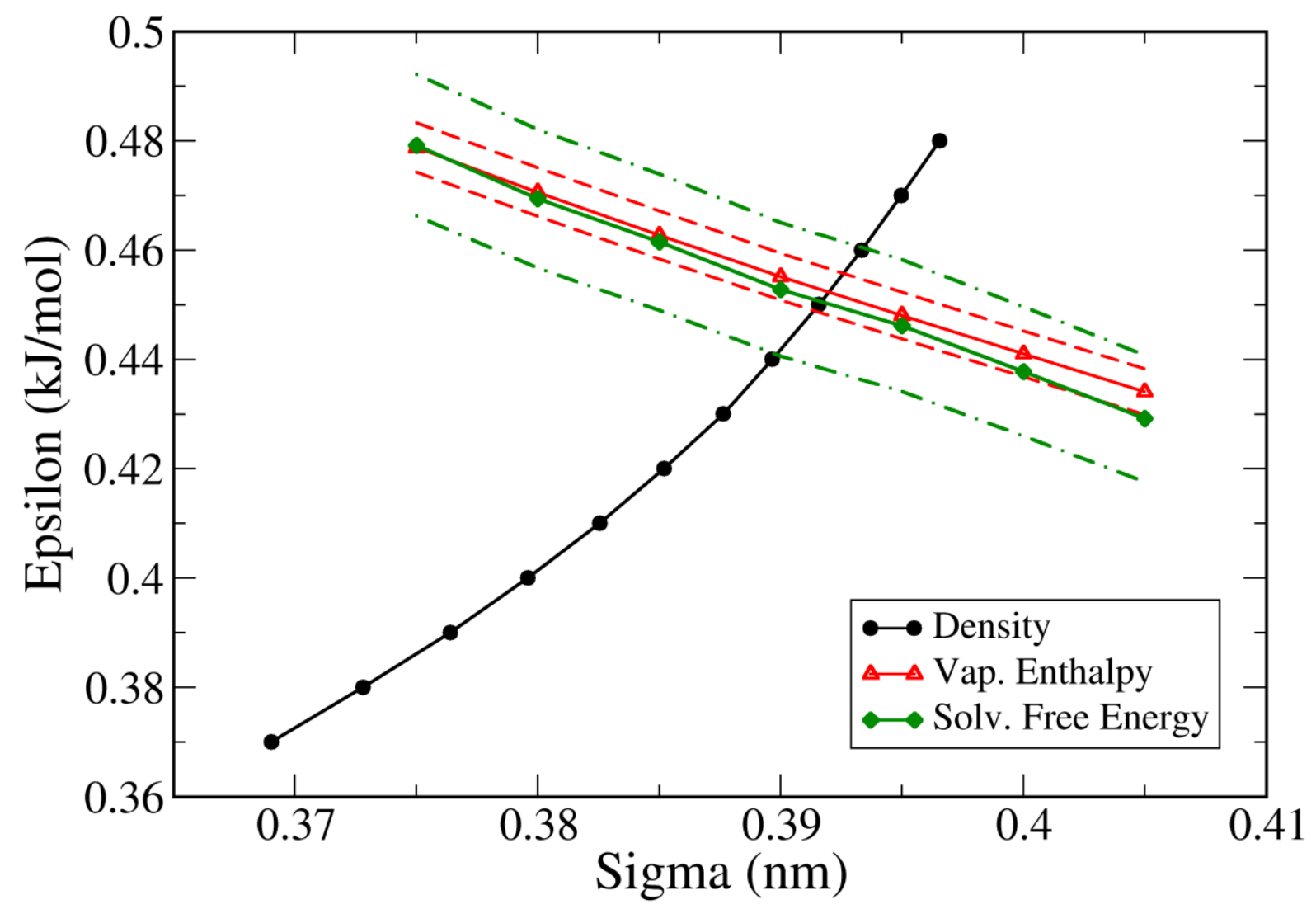

Figure 4 - Determination of optimal set of parameters for cyclohexane. The full lines show the paths in parameter space that provide an accurate match for density (filled circles and black line), enthalpy of vaporization (open triangles and red line) and self-solvation free energy (filled diamonds and green line). The dashed lines represent the estimated uncertainty in the experimental reference values (dashed red lines for enthalpy of vaporization and dotted-dashed green lines for self-solvation free energy).

To end this section, it is worth recalling that the parameters of the new model were optimized to match density and solvation free energy at room temperature. It is not evident $a$ priori that those parameters are transferrable to different temperatures. Figure S1 compares the simulated density using the new model parameters against experimental data for liquid cyclohexane as a function of temperature within a relatively wide range. As we can see, the model accurately predicts the density in the entire temperature range. Although further tests would need to be carried out to determine if there is any loss of performance for other properties (namely solvation free energies), the level of agreement for density is certainly encouraging.

\section{2 - Linear and Branched Alkanes}

In this section, a correction is implemented to the original TraPPE linear and branched alkane parameters in order to provide a better match against both density and solvation free energies, using as a reference the same experimental dataset [18, 19]. As previously, the bonded parameters of the original TraPPE model were adopted, and the same atom types for linear and branched molecules were maintained, i.e., $\mathrm{CH}_{4}, \mathrm{CH}_{3}, \mathrm{CH}_{2}, \mathrm{CH}$, and $\mathrm{C}$ groups. After statistical analysis of the TraPPE predictions for all the solute-solvent pairs considered, 
there was nothing to indicate that the deviations from experiment were due to a particular set of parameters. Instead, deviations were practically independent of the type of sites present in the solute and solvent molecules. Based on these observations, it was decided to simply rescale the values of $\sigma$ and $\varepsilon$ for all atom types simultaneously (except $\mathrm{CH}_{4}$, see below) by a constant factor - one scaling factor for $\sigma$ and another for $\varepsilon$. This greatly simplified the parameterization procedure while still bringing significant improvements in performance over the entire range of molecular architectures, as will be shown later. It should be noted, however, that this approach only makes sense because one already has an initial guess of parameters that is quite close to the optimum (i.e., the original TraPPE parameters). Were this not the case, and the usual approach of parameterizing each atom type separately would have to be adopted.

The appropriate scaling factors for $\sigma$ and $\varepsilon$ were determined by making use of the observed variation of $\rho$ and $\Delta G$ with those parameters for cyclohexane self-solvation (Figures 1 and 3). In short, the average gradient of change of each property with each parameter was calculated and then used to estimate the necessary percent change in $\sigma$ and $\varepsilon$ that would be necessary to bring the simulation predictions into agreement with experiment. More precisely, it was estimated that increasing $\sigma$ by $1 \%$ and increasing $\varepsilon$ by $2 \%$ would cause $\Delta G$ to increase in magnitude (i.e., become more negative) by about $6 \%$ and $\rho$ to decrease by about $1 \%$. The solvation free energy of one solute/solvent pair was then calculated with the rescaled parameters to test the actual improvement achieved. Nonane in hexadecane was selected as the training set because this corresponded to one of the largest magnitudes of $\Delta G$, and because the relative error for the TraPPE model turned out to be nearly identical to the average relative error of the entire data set, so a good match for this pair is a good indicator for overall agreement with experiment. Although it was expected that more than one iteration would be needed, this was not the case - the first guess of the correction factor turned out to yield excellent agreement for the solvation free energy of nonane in hexadecane. Once again, this was most likely due to the already good performance of the original TraPPE parameters.

Figures 5, 6, and S1 show how the new parameters (Table 1) lead to an excellent match between simulation and experiment for linear alkanes dissolved in other linear alkanes. In particular, the self-solvation of linear alkanes (Figure 6) is in almost perfect agreement with experiment, which as discussed previously [17] suggests that the vapor pressure of the pure liquids is also predicted accurately. Moreover, both the density and the enthalpy of vaporization of pure linear alkane liquids are more accurately predicted by the new model than by the original TraPPE force-field (Figure 7). The new model is also able to qualitatively and quantitatively predict the effect of an increase in chain length of the solvent (Figure S2) and of the solute (Figure 5). Improvements are also significant for linear solutes dissolved in branched (Figure S3) and cyclic (Figure S4) solvents, as well as for solvation of branched solutes (Figures S5 and S6). 


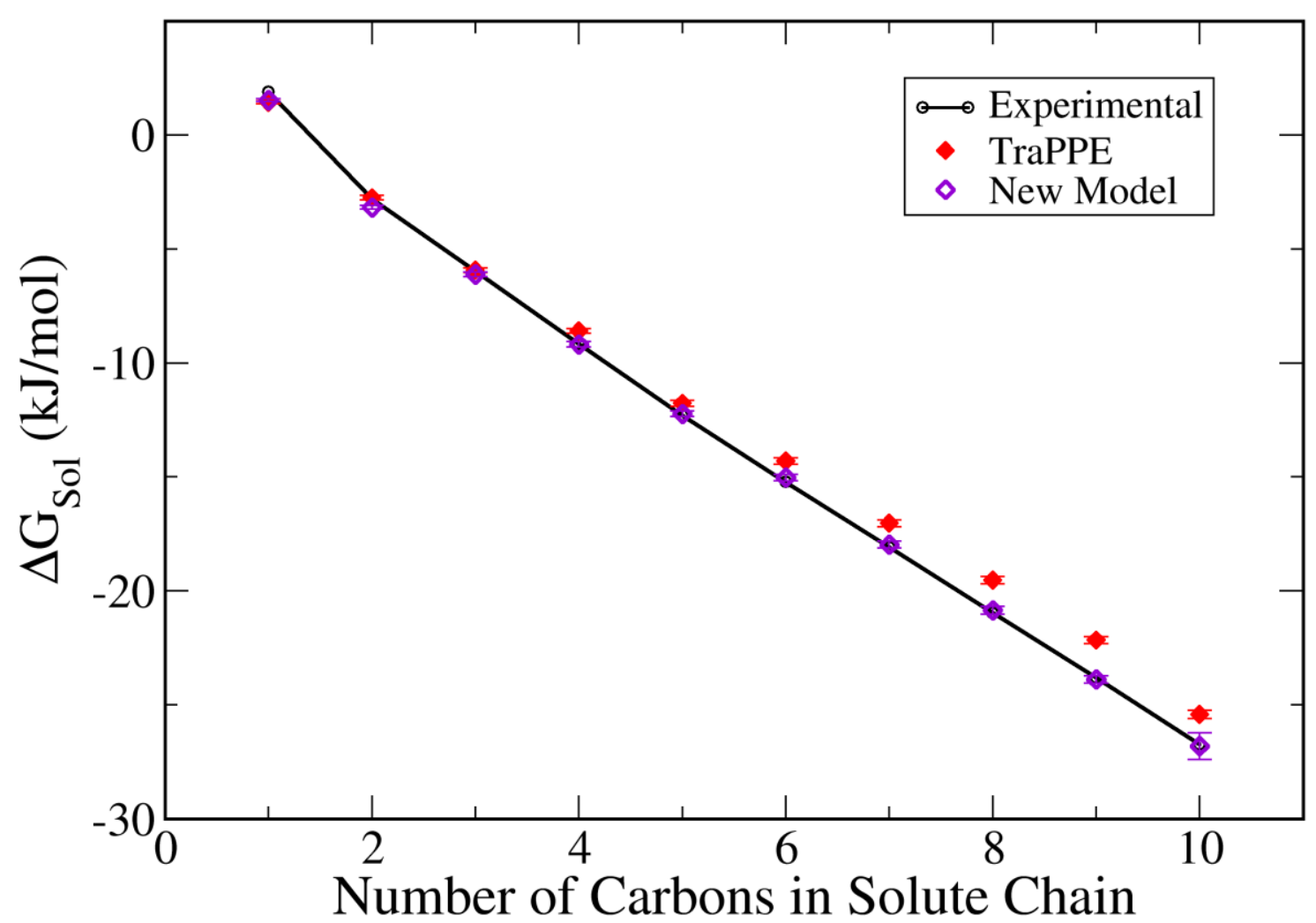

Figure 5 - Comparison between the original TraPPE model and the new model for linear alkane solutes of different chain length in n-hexadecane solvent.

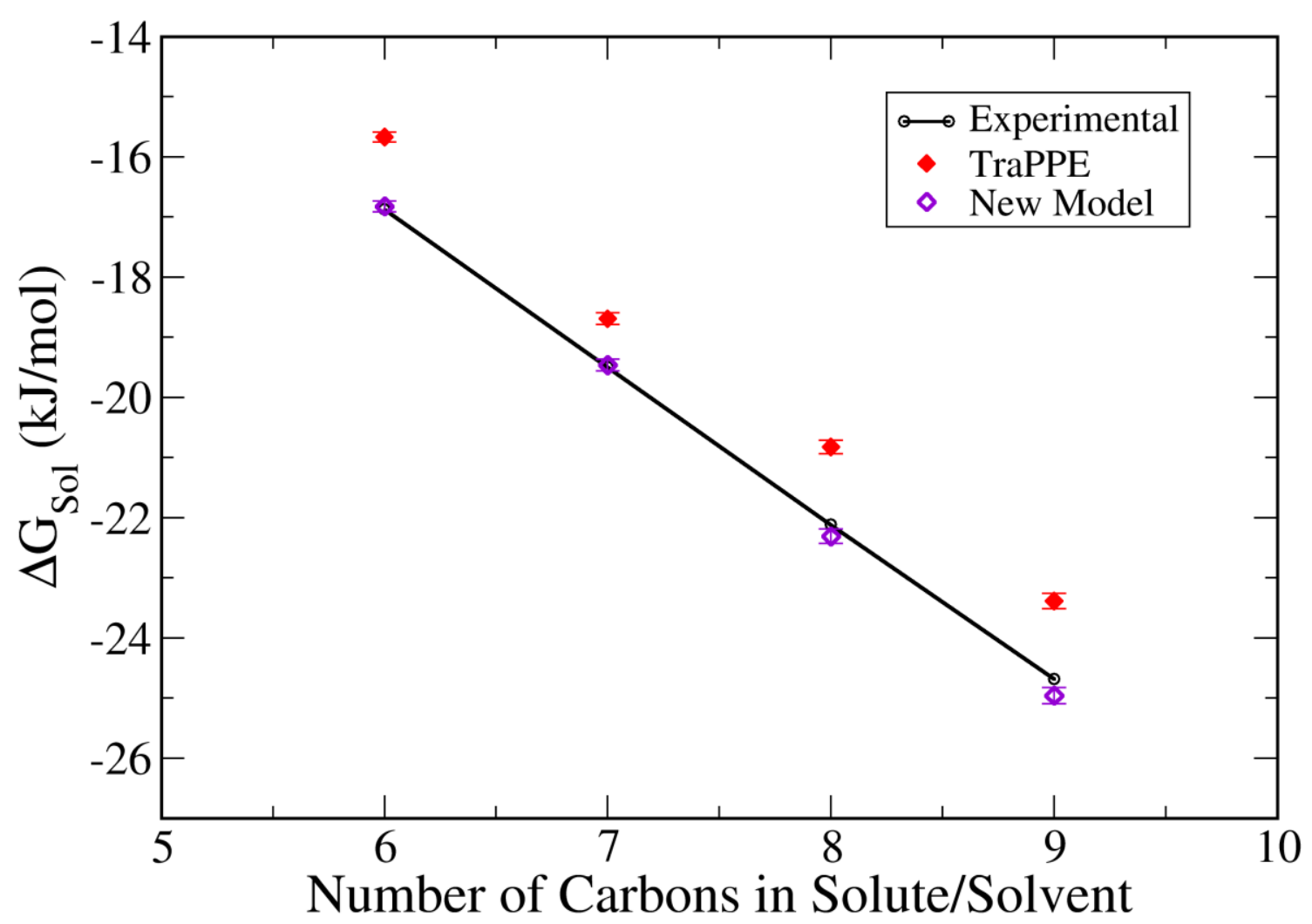

Figure 6 - Comparison between the original TraPPE model and the new model for linear alkane self-solvation (solute and solvent are the same molecule). 

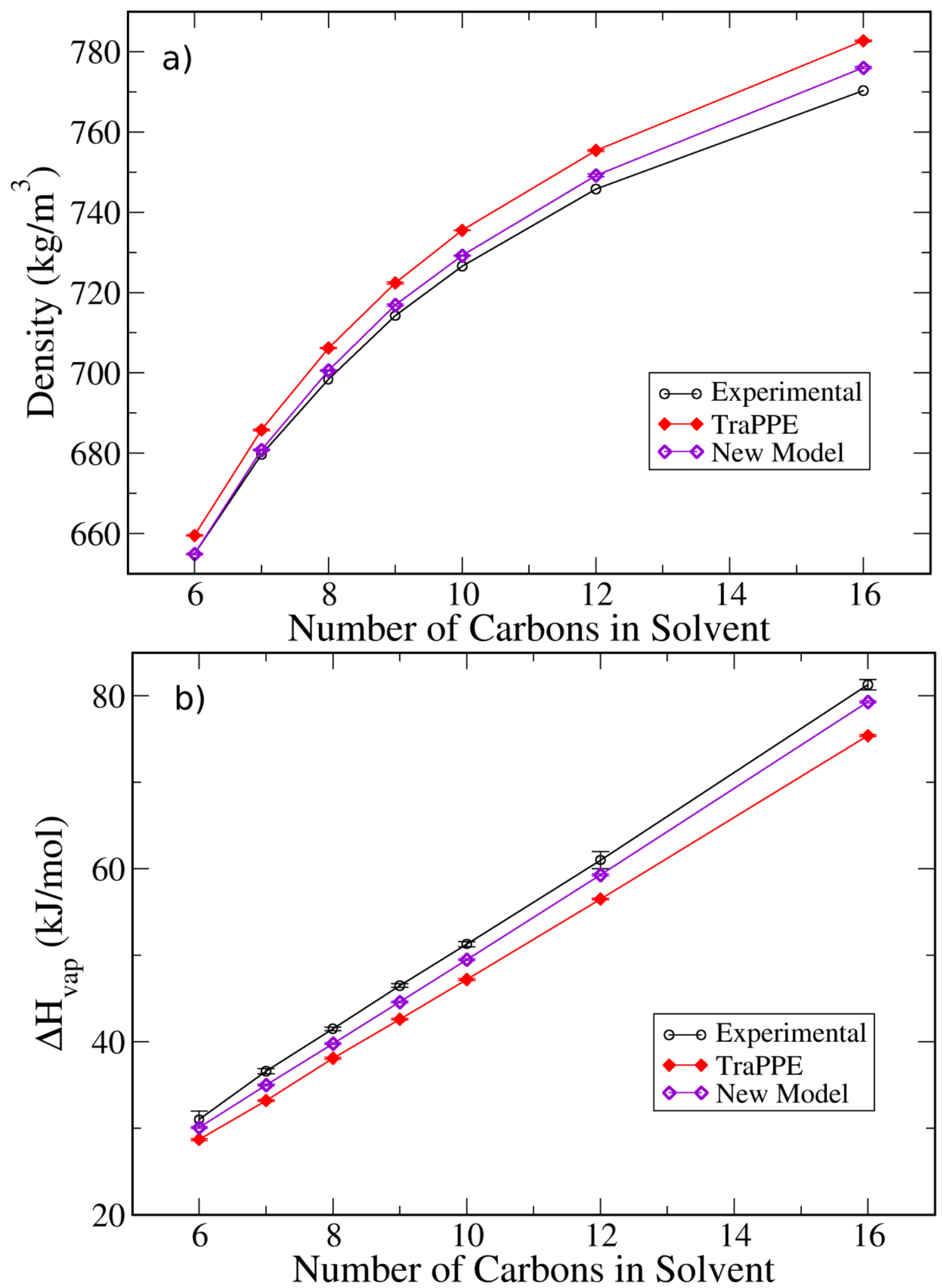

Figure 7 - Comparison between the original TraPPE model and the new model for the density (a) and enthalpy of vaporization (b) of pure alkanes of increasing chain length.

Figure 8 shows the comparison between the new model and TraPPE for cyclic alkane solutes in n-hexadecane solvent. As discussed in the first paper of this series [17], the use of 
separate parameters for each cyclic alkane in TraPPE leads to a trend which is significantly different from the experimental data (e.g., the solvation free energy of cyclopentane is almost the same as that of cyclohexane). This observation was the main reason that led to the choice of using a single atom type for cyclic $\mathrm{CH}_{2}$ groups in the new model. As we can see, the chosen approach leads to a trend that is much closer to experiment. The adjustment of the LJ parameters also leads to excellent quantitative agreement for all solutes except cyclopentane (recall that the cyclic $\mathrm{CH}_{2}$ parameters were designed to match the self-solvation free energy of cyclohexane, as described in section 3.1). Although a better match for cyclopentane could have potentially been obtained by introducing a separate set of parameters for $\mathrm{CH}_{2}$ groups in this molecule, this would increase the complexity of the model beyond what is felt as justifiable. Finally, Figure 9 shows that the new model is now able to quantitatively predict the solvation free energy of different isomers of hexane, therefore it can correctly describe rather subtle effects of molecular architecture on solvation (the reader is referred to the first paper of this series for a detailed explanation of the degree of branching classification [17]).

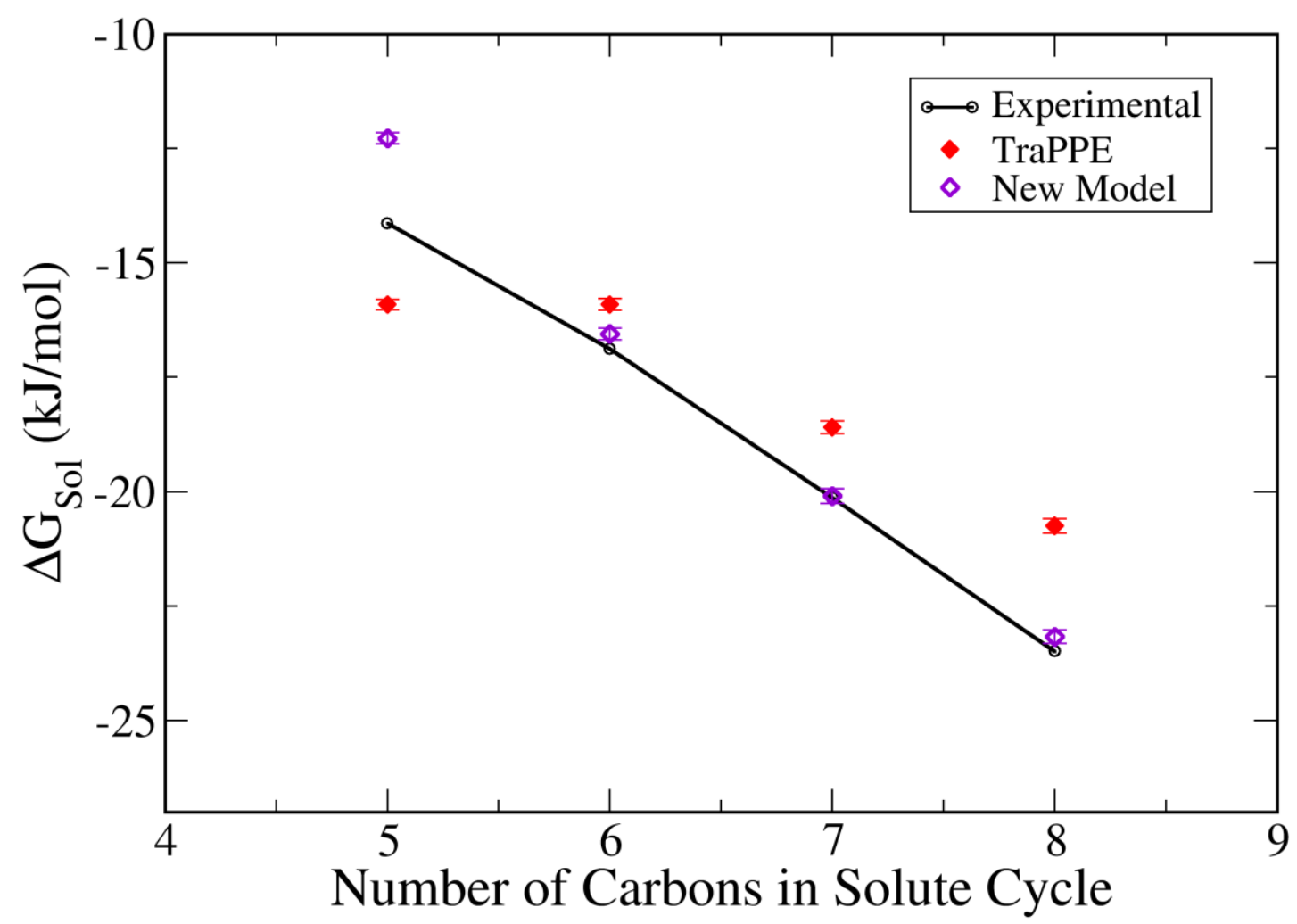

Figure 8-Comparison between the original TraPPE model and the new model for solvation of cyclic alkane solutes of different size in n-hexadecane solvent. 


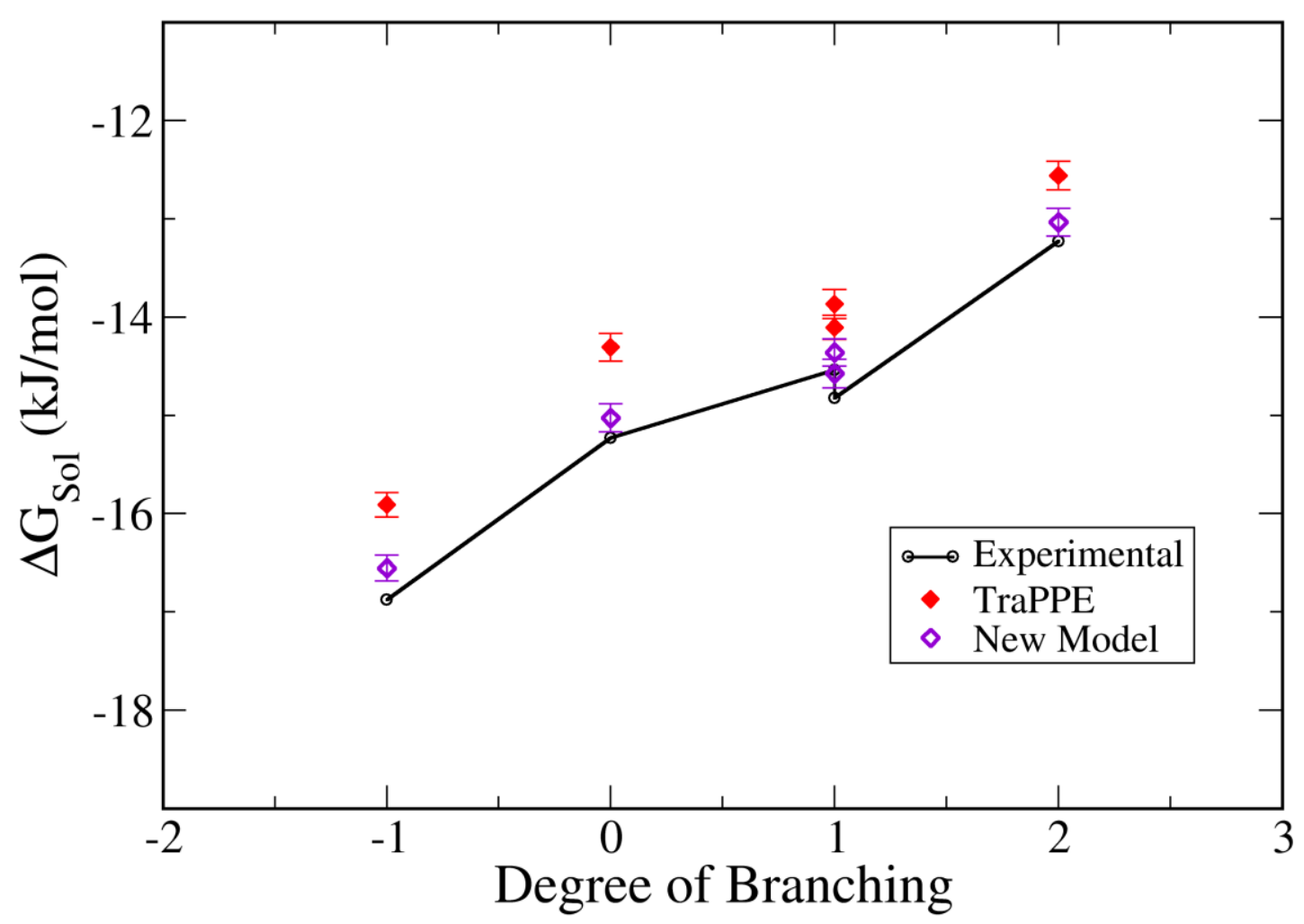

Figure 9-Comparison between the original TraPPE model and the new model for solvation of hexane isomers of different degree of branching $(D o B)$ in n-hexadecane solvent. The DoB is 0 for linear molecules, 1 for single-branched molecules, 2 for double-branched molecules and, rather arbitrarily, -1 for cyclic molecules (see [17] for details).

For all the atom types discussed until now, both $\sigma$ and $\varepsilon$ had to be increased relative to the original TraPPE model to obtain good agreement with experimental solvation free energies. Methane, however, is an exception - TraPPE actually overestimates the degree of solvation (i.e., $\Delta G_{\text {sol }}$ is less positive than experiment; see Table 1 of the previous paper [17]), which goes in the opposite direction of the general trend. This means that methane requires a separate specific parameterization effort. The experimental database of Katritzky et al. [18, 19] contains only a single point for methane (in n-hexadecane), which was considered insufficient to provide a robust set of parameters. As such, additional data from Wolfenden et al. [34] for methane solvated in cyclohexane was used. The new parameters for methane were determined by simultaneously matching the experimental solvation free energy in cyclohexane and the density of pure methane at its standard boiling point [37], and the solvation free energy in n-hexadecane was then used for validation of the parameters. Making use of the trends depicted in Figure 3 for cyclohexane, it was concluded that to match the solvation free energy a decrease in both $\sigma$ and $\varepsilon$ was needed. One started by decreasing $\sigma$ by an initial amount, then found the corresponding value of $\varepsilon$ that provided a close match to the pure fluid density (iterating in density is more efficient, as the simulations are considerably faster). This pair of parameters was then tested against the free energy, and a new guess for $\sigma$ was obtained by linear interpolation (i.e., assuming a linear variation of solvation free energy with both parameters, as shown in Figure 3). The parameters, shown in Table 1, converged 
after two iterations. The new parameters lead to very good agreement with the experimental solvation free energies (absolute deviations of $-0.044 \mathrm{~kJ} / \mathrm{mol}$ for methane in cyclohexane and $0.139 \mathrm{~kJ} / \mathrm{mol}$ in n-hexadecane) and pure methane density (absolute deviation of $0.4 \mathrm{~kg} / \mathrm{m}^{3}$ ).

To conclude the analysis for alkanes, Figure 10 shows an overall comparison between experiments and simulations using the new adjusted united-atom model for the entire set of alkane solute-solvent pairs. Overall statistics are provided in Table 2, in comparison with the original TraPPE model (for the performance of other UA models, the reader is referred to Table 2 of the first paper of this series [17]). As can be seen, agreement between simulation and experiment is excellent across all types of alkane molecules. The relative deviation is about $1 \%$, while the RMSD is $0.52 \mathrm{~kJ} / \mathrm{mol}$, which is within the order of uncertainty in the experimental data [40].

Table 2 - Measures of deviation between experimental data and simulations using different models, for the entire alkane data set analyzed: $M S D=$ mean signed deviation; $R M S D=$ root mean squared deviation.

\begin{tabular}{|l|c|c|}
\hline & TraPPE & New Model \\
\hline Slope (fit) & 0.940 & 1.001 \\
\hline $\mathrm{R}^{2}$ (fit) & 0.986 & 0.992 \\
\hline MSD (kJ/mol) & -0.967 & -0.020 \\
\hline RMSD (kJ/mol) & 1.204 & 0.511 \\
\hline
\end{tabular}

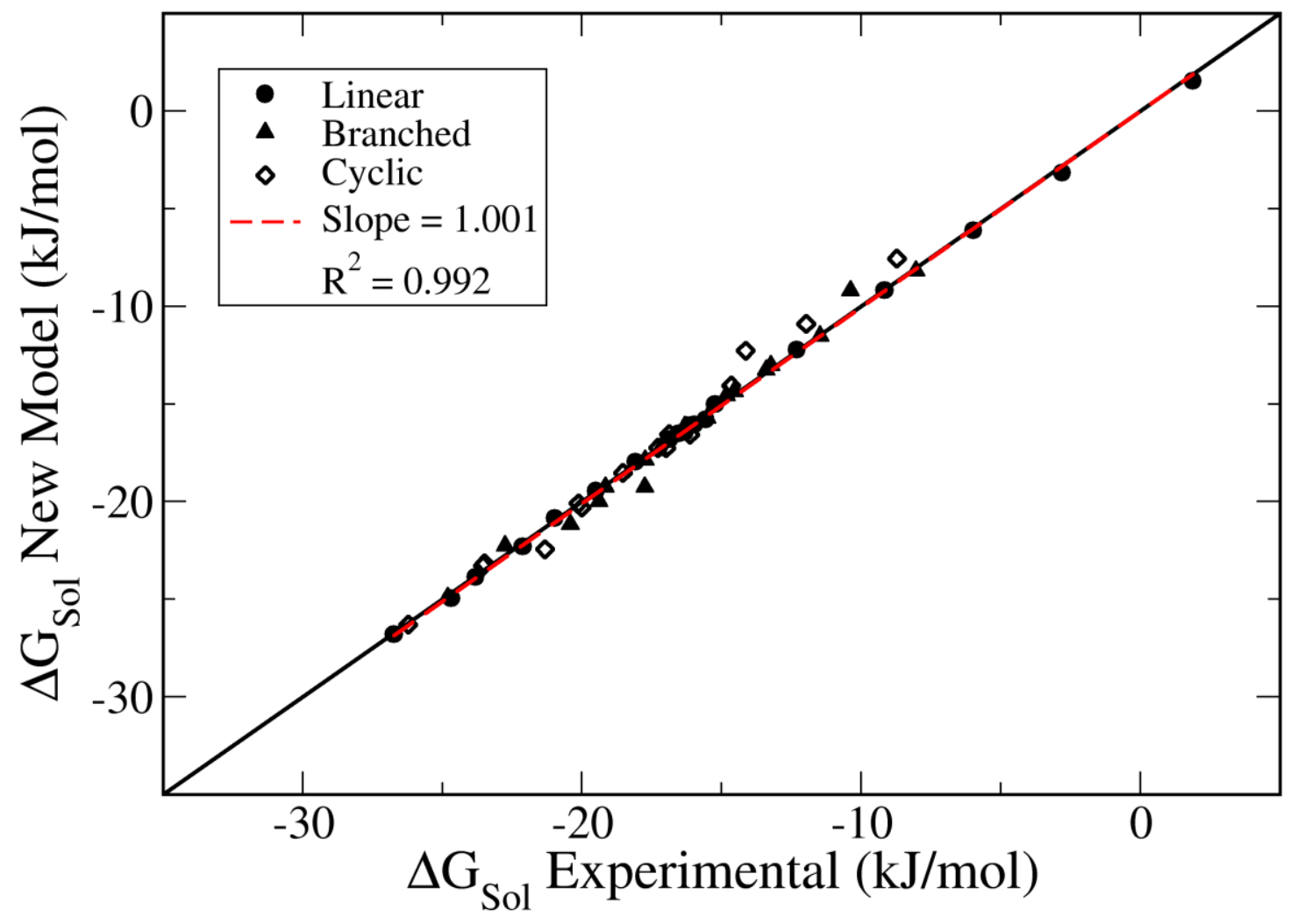

Figure 10 - Comparison between experimental and simulated solvation free energies for the entire alkane data set using the newly developed force-field. The dashed red line shows a linear fit (with forced intercept at the origin) through the data. The slope and the correlation coefficient of the fit are also reported. 


\section{3 - Alkenes and Alkynes}

After establishing that the new model can predict solvation free energies of alkanes to a high degree of accuracy, the same approach is extended to alkene and alkyne molecules. Fewer experimental data points $[18,19]$ are available for those molecules, particularly for the latter, but these are nevertheless sufficient. As a first approach, the original TraPPE parameters were tested for solvation of alkane solutes in alkene solvents. The same systematic overestimation of solvation free energies was observed when the solvent was 1octene (Figure 11) or 1-decene (Figure S7). However, when the new parameters, optimized in section 3.2, were used for the alkane solutes, the predictions very accurately reproduced the experimental data. This suggests that the original TraPPE parameters for alkene groups [42] provide a good enough representation of pure alkene solvents (although, as described above, the alkane group parameters required a correction). To confirm this, the density and enthalpy of vaporization of pure alkene liquids were compared against experimental data in Figure 12. As we can see, when the TraPPE alkane parameters are corrected, but the alkene parameters are kept the same, both properties of alkene solvents are reproduced very accurately.

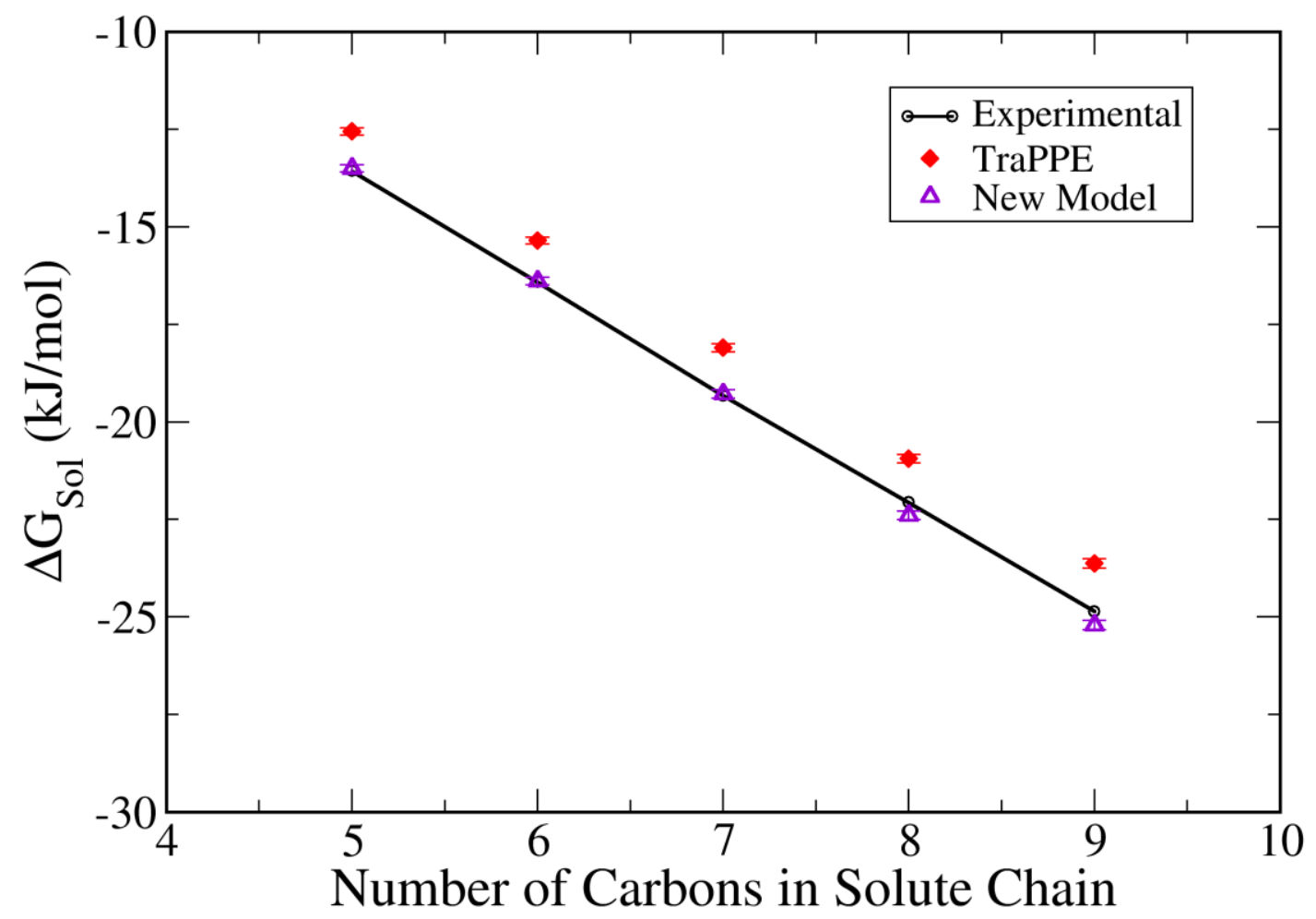

Figure 11 - Comparison between the original TraPPE model and the new model for linear alkane solutes of different chain length in 1-octene solvent. 

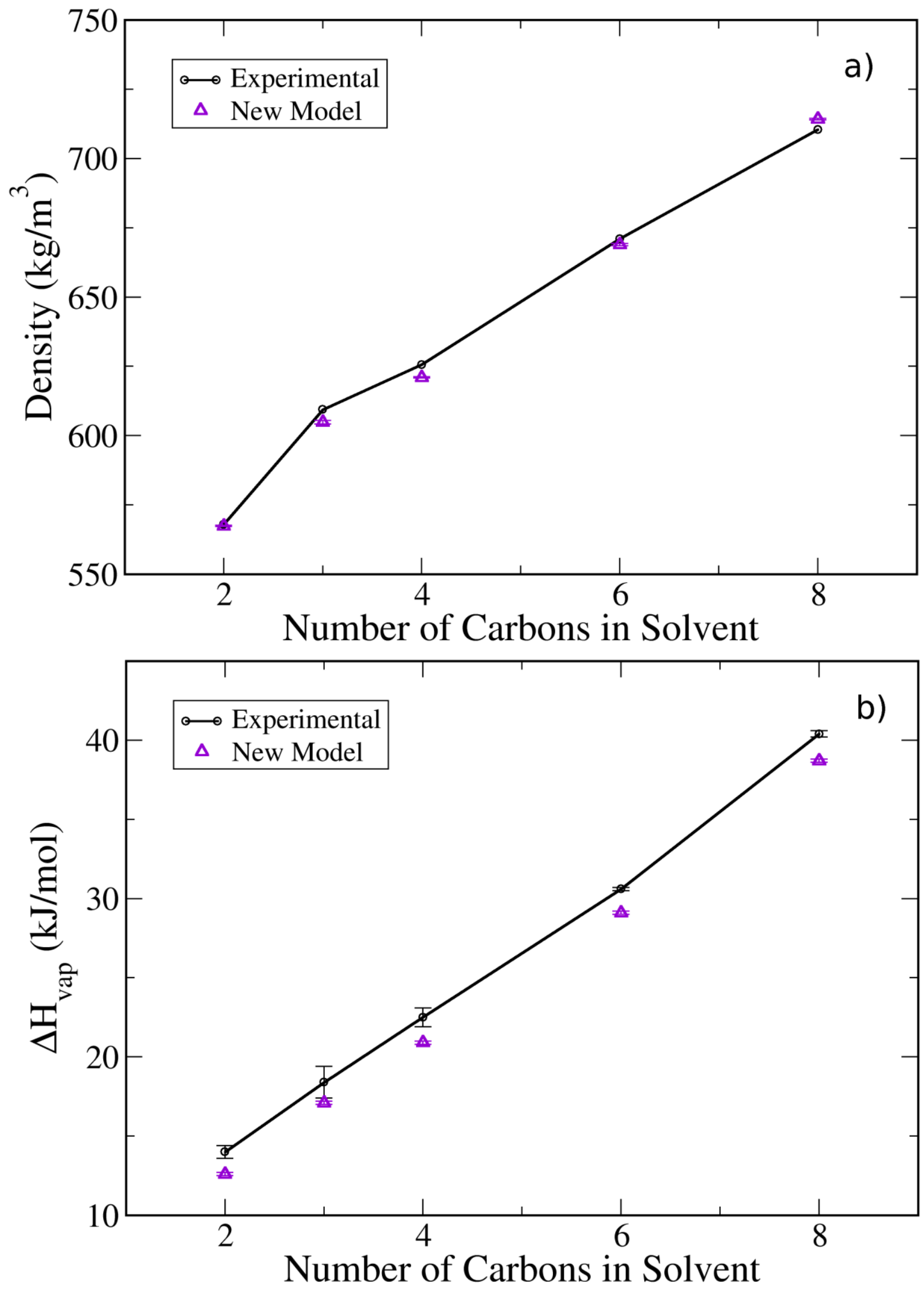

Figure 12 - Comparison between experimental data [37, 38] and predictions of the new model for the density (a) and enthalpy of vaporization (b) of pure alkenes of increasing chain length. Data are at $298 \mathrm{~K}$ except for ethene, propene and 1-butene, which were measured at their respective boiling points at 1 bar. 
As discussed previously, a much more stringent test of model parameters is to predict solvation free energy of alkene solutes. In Figures 13 and S8, predictions of the TraPPE model [42] as well as the improved model are compared against experimental data for linear alkene solutes in $\mathrm{n}$-heptane and $\mathrm{n}$-hexadecane solvents, respectively. Interestingly, the predictions of the original TraPPE model (i.e., with uncorrected alkane parameters) are quite close to experiment, although a slight systematic overestimation can be observed for larger solute molecules. In fact, when predictions of the original TraPPE model are compared against experimental data for the entire dataset involving alkenes, as either solutes or solvents (Figure S9), we see the same systematic overestimation reported in the previous paper of this series for alkanes [17] - solvation free energies are consistently more positive than experiment - but with a smaller magnitude of deviation (about 4-5\% compared to $6 \%$ for pure alkanes). This again suggests that the deficiencies of the TraPPE model are mostly due to the alkane parameters and not to the alkene parameters.

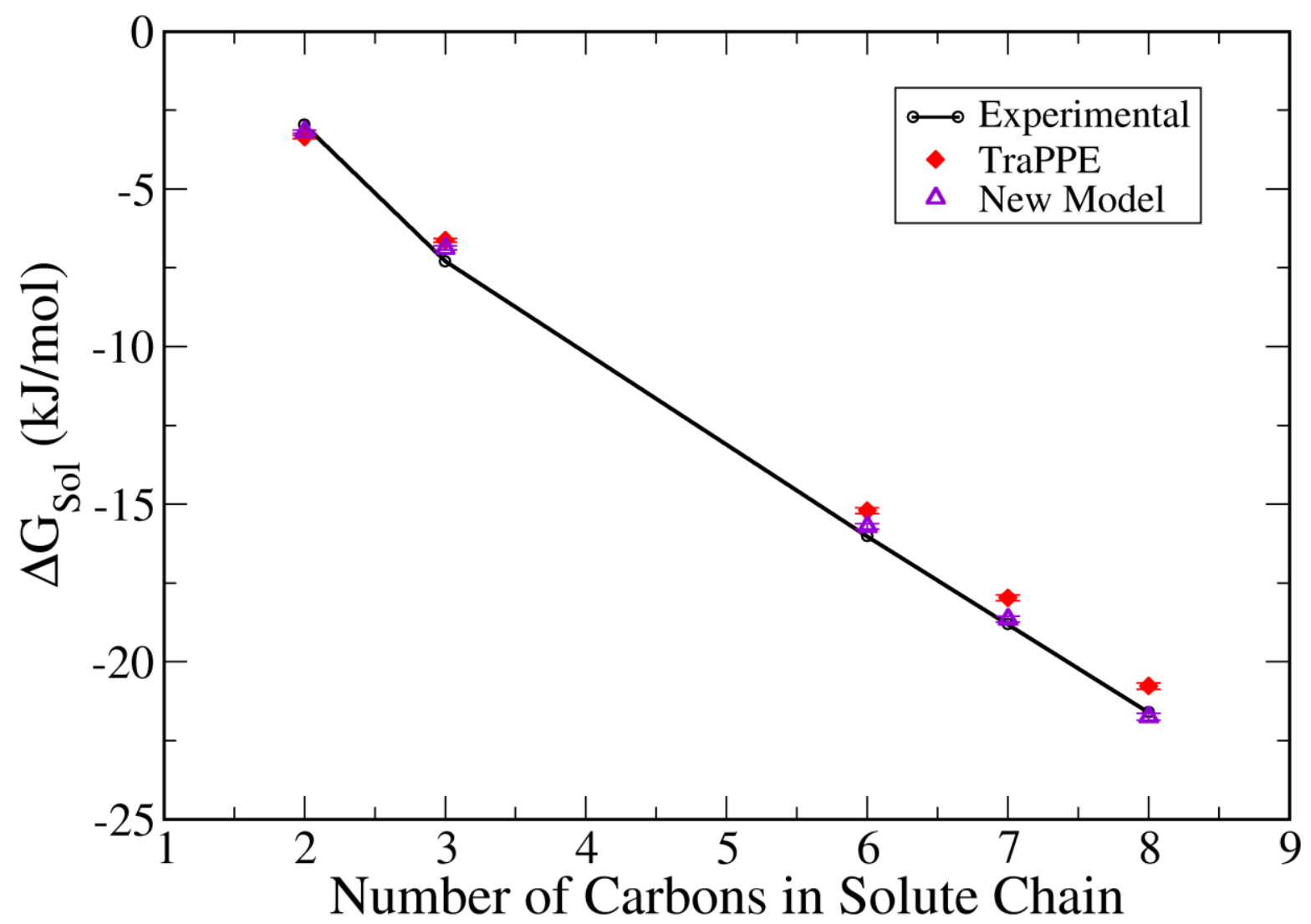

Figure 13 - Comparison between the original TraPPE model and the new model for linear alkene solutes of different chain length in n-heptane solvent.

When the alkane parameters are corrected to the values determined in sections 3.1 and 3.2, keeping the alkene parameters the same as in the original TraPPE model, predictions of alkene solvation are excellent. The exception is solvation of 1-butene in n-hexadecane (Figure S8), but this is expected to be an error in the experimental data [18, 19], as this point completely departs from the expected linear trend. In fact, the corresponding value reported in the Minnesota Solvation Database [35] is $-8.49 \mathrm{~kJ} / \mathrm{mol}$, which fits within the linear trend 
and agrees very well with the new model's predictions (absolute deviation of $-0.102 \mathrm{~kJ} / \mathrm{mol}$ ). The excellent agreement obtained with the original TraPPE alkene parameters confirms that no correction to these parameters is needed. As such, the original parameters for alkene groups were maintained in the new solvation model (Table 1).

The final stage of the new model development was to examine solvation free energies involving alkynes. Although the polarity of hydrocarbons increases as they become less saturated, leading some alkenes and alkynes to develop a small dipole moment, a neutral UA approach was still adopted. Testing the validity of this approximation for solvation in polar solvents will be the subject of future work. Perhaps surprisingly, it was not possible to find any UA models of alkynes in the literature (parameters exist for all-atom models, but these contain explicit hydrogens and point charges, so they are not suitable for our purposes). Therefore, a new parameter set was developed from scratch, aiming to reproduce solvation free energies and pure liquid densities of alkyne molecules.

The first step was to determine parameters for $\mathrm{CH}$ groups with $s p$ hybridization by matching the experimental solvation free energy of acetylene in n-heptane $[18,19]$ and the density of pure acetylene at its standard boiling point [37]. The parameterization strategy was very similar to the one described above for methane (section 3.2), except that here one did not have a good initial guess for the parameters. The line in $(\sigma, \varepsilon)$ parameter space that provided a good match to the experimental density of acetylene (i.e., the analog of the black line in Figure 4) was first traced, given that density calculations are computationally cheap. This focused on a range of $\sigma$ values between $0.36 \mathrm{~nm}$ and $0.32 \mathrm{~nm}$, as the value of this parameter is expected to decrease as carbon hybridization increases [11, 42]. Two points on this line were then selected and two solvation free energy calculations were performed for those pairs of parameters. Comparing these two results to the target experimental value, a new estimate of $\sigma$ was obtained by linear extrapolation (i.e., assuming linear dependences of free energy with each of the parameters, as observed in Figure 3 ). The optimal value of $\varepsilon$ corresponding to that value of $\sigma$ was then obtained by matching the experimental acetylene density, and the cycle was repeated until convergence. Three iterations were sufficient to obtain the converged set of parameters shown in Table 1. The validity of the new parameters was assessed by predicting the solvation free energy of acetylene in n-hexadecane, for which the deviation was only $0.25 \mathrm{~kJ} / \mathrm{mol}$ (i.e., well within the precision of experimental data).

Once the $\mathrm{CH}$ parameters were found, one moved on to parameterize the $\mathrm{C}(s p)$ group. The experimental database only contained solvation free energies for propyne, 1-butyne, 1pentyne and 1-hexyne in n-hexadecane. It was decided to tune the $\mathrm{C}(s p)$ parameters to simultaneously match the density of 1-hexyne and the solvation free energy of propyne in nhexadecane. The strategy adopted was identical to the one described above for the $\mathrm{CH}$ group, and converged after three iterations. The quality of the parameters was tested against solvation free energies, densities and enthalpies of vaporization of the other alkynes. It is clear from Figure 14 that the new set of parameters yields solvation free energies for the 
whole alkyne series in very good agreement with experimental data, which is the main purpose of the new model. Agreement for density is also good (see Figure 15a) except for 1butyne, which shows a deviation of $6.6 \%$, much higher than for any other solvent tested in this work. Although at present no definitive explanation for this unusual result can be provided, it is noteworthy that the uncertainties in the density calculations for alkynes larger than acetylene are quite high. As discussed in section 2 , the $180^{\circ}$ angle in those molecules led to unphysical molecular distortions in MD runs with a stochastic dynamics integrator. Although this problem was subsequently solved, it may have still led to the observed large amplitude fluctuations in the density of the pure alkynes, and concomitantly large uncertainties.

Finally, it can be seen in Figure 15b that the enthalpies of vaporization of the alkyne liquids are systematically underestimated. Although absolute deviations are not very large, their systematic nature may represent an inherent limitation of the united-atom approach for alkynes. Further work is necessary to fully ascertain this. Arguably, it may have been possible to tune the parameters for $\mathrm{CH}$ and $\mathrm{C}$ groups simultaneously to provide the best compromise in fitting the densities, enthalpies of vaporization and solvation free energies for all the molecules studied. However, because the target experimental data is quite limited and because of the technical issued discussed above, this was not pursued any further.

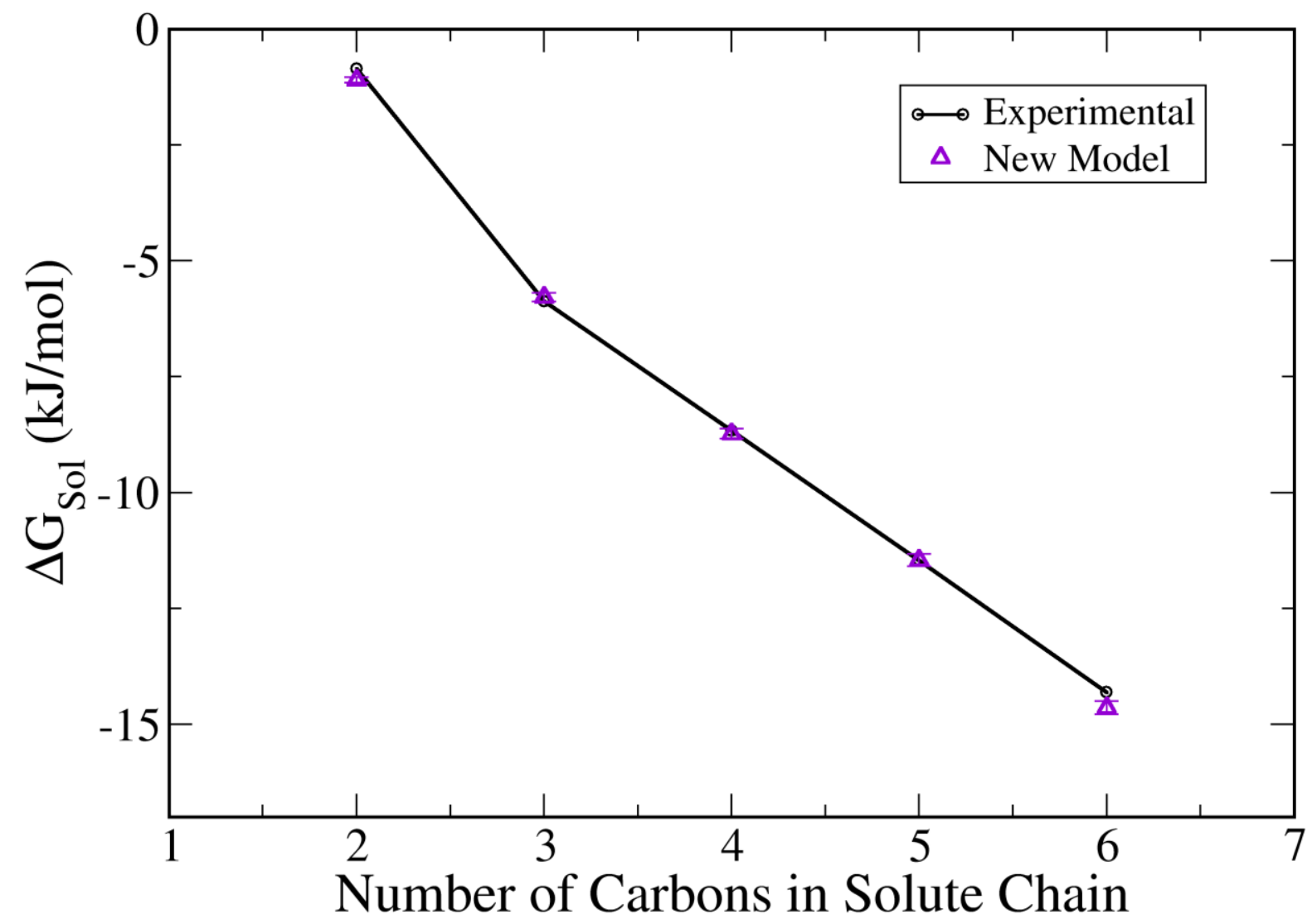

Figure 14 - Comparison between experimental data and predictions of the new model for linear alkyne solutes of different chain length in n-hexadecane solvent. 

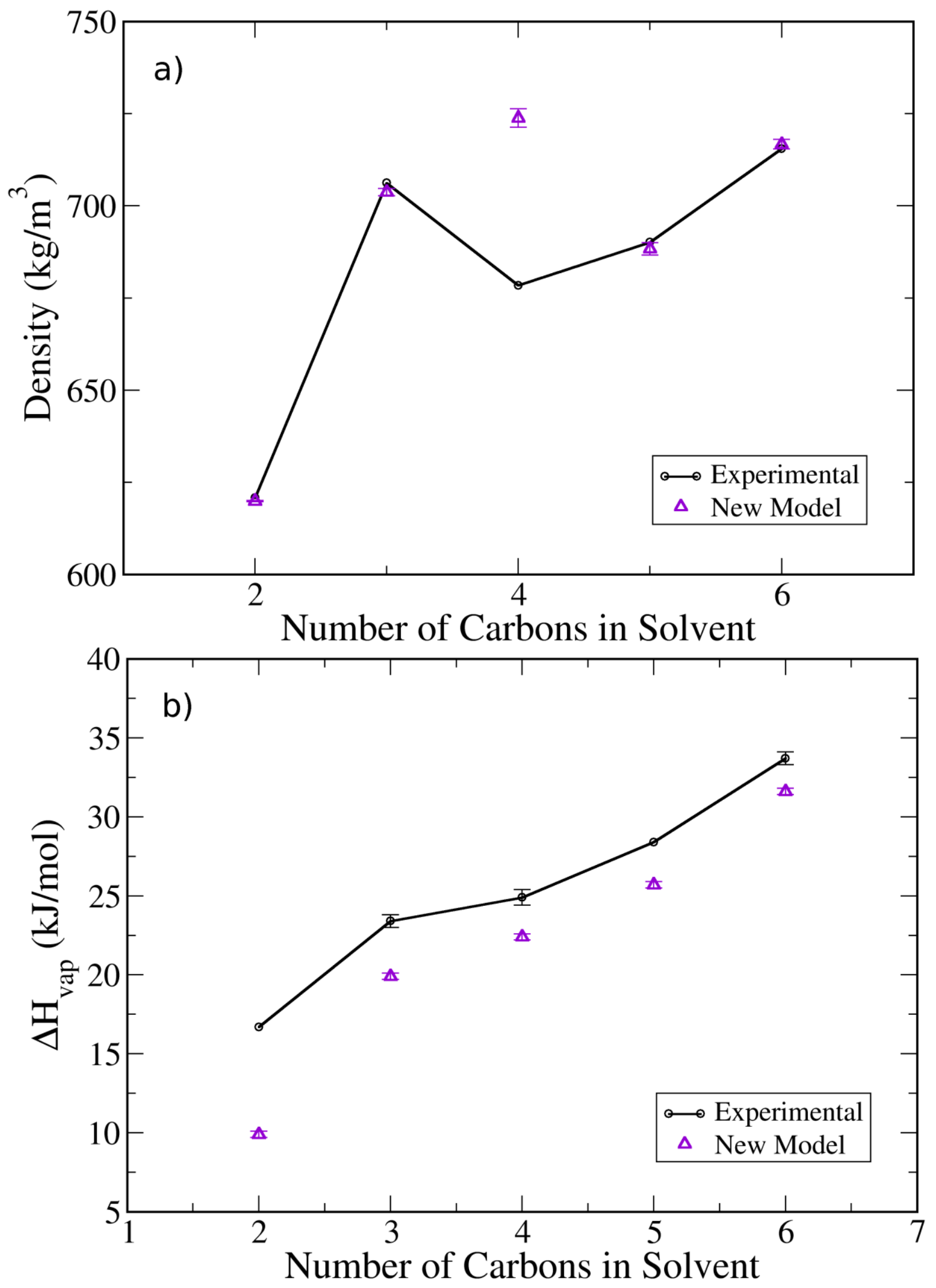

Figure 15 - Comparison between experimental data [37, 38] and predictions of the new model for the density (a) and enthalpy of vaporization (b) of pure alkynes of increasing chain length. Data are at $293 \mathrm{~K}$ except for acetylene, propyne and 1-butyne, which are at their respective boiling temperatures. 
Figure 16 compares the predictions of the new model against experimental data for the entire data set involving alkenes and alkynes $[18,19]$ (which also contain alkane groups, as discussed above). It is clear that the new model yields predictions in excellent agreement with experimental data for the entire dataset, with the exception of two outliers: 1-butene in n-hexadecane and 1-pentene in 2,2,4-trimethylpentane. The former was discussed above and is believed to be an error in the experimental data (the value reported in the Minesotta Solvation Database [35] is actually much closer to our predictions). For the latter, however, the Minesotta Solvation Database [35] reports a value $(-9.87 \mathrm{~kJ} / \mathrm{mol})$ that is almost identical to that of Katritzky et al. [18, 19]. At present, the origin of this discrepancy is not completely understood, and further tests (both experimental and theoretical) are required. Overall, even including the two outliers, the mean signed deviation from experimental data is $0.17 \mathrm{~kJ} / \mathrm{mol}$, corresponding to a relative deviation of about $1 \%$, and the RMSD is only $0.68 \mathrm{~kJ} / \mathrm{mol}$, again well within the experimental uncertainty [40].

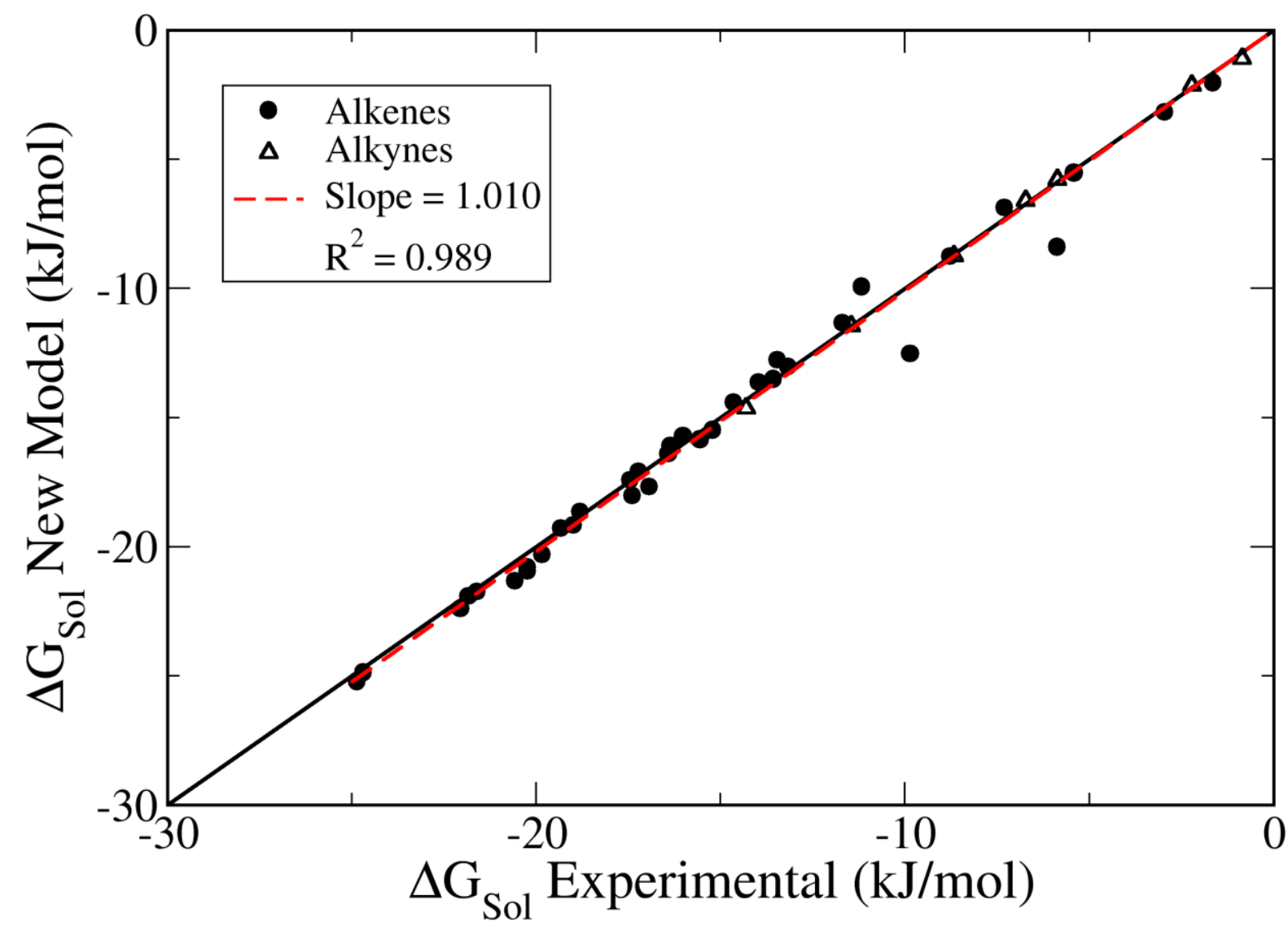

Figure 16 - Comparison between experimental and simulated solvation free energies for the entire alkene and alkyne data set using the newly developed force-field. The dashed red line shows a linear fit (with forced intercept at the origin) through the data. The slope and the correlation coefficient of the fit are also reported. 


\section{4 - Conclusions}

In this paper a new fully transferrable united-atom model for hydrocarbon molecules that is able to accurately predict hydrophobic solvation free energies (i.e., solvation of hydrocarbons in other hydrocarbons) has been presented. The starting point for the parameterization was the TraPPE force-field, as it has been shown in the previous paper of this series that it performs best among several popular UA models. Accurate solvation free energy predictions of linear and branched alkanes were obtained by implementing a small correction to the original TraPPE parameters for $\mathrm{CH}_{3}, \mathrm{CH}_{2}, \mathrm{CH}$ and $\mathrm{C}$ sites with $s p^{3}$ hybridization (increasing $\sigma$ by $1 \%$ and $\varepsilon$ by $2 \%$ ). Methane parameters, however, required a small correction (below $1 \%$ for both $\sigma$ and $\varepsilon$ ) in the opposite direction. A new set of parameters for $\mathrm{CH}_{2}$ groups in cyclic alkanes that is applicable to all molecules of this type has also been developed. These changes were able to correct the systematic underestimation of alkane solvation free energies observed for the TraPPE model, while simultaneously yielding a better description of pure fluid densities. The new alkane parameters also led to excellent predictions of alkene solvation free energies, when combined with the original TraPPE parameters for $\mathrm{CH}_{2}\left(s p^{2}\right)$ and $\mathrm{CH}\left(s p^{2}\right)$ sites. For this reason, the parameters for $s p^{2}$ sites were kept unchanged in the new model. Finally, a new set of parameters for sites with $s p$ hybridization has been proposed, which led to accurate predictions of solvation free energies and densities of alkynes. Averaging over the entire data set comprising 95 solute/solvent pairs, the mean signed deviation between experiments and simulations using the new model is $0.064 \mathrm{~kJ} / \mathrm{mol}$, while the RMSD is only $0.6 \mathrm{~kJ} / \mathrm{mol}$. The latter is below the estimated uncertainty of $0.8 \mathrm{~kJ} / \mathrm{mol}$ in the experimental measurements. This new set of parameters represents an improvement over previous models and is a solid base for development of a classical non-polarizable force-field that is able to accurately predict solvation free energies in both polar and non-polar solvents. Extension of this model to describe polar compounds requires, of course, consideration of electrostatic interactions. Further work in this direction is currently underway.

\section{Supplementary Material}

Additional results figures, as detailed in the main text; full table with all experimental and simulated solvation free energies, full tables of interaction parameters of the new model. Input files for all solvation free energy calculations are freely available from the University of Strathclyde's data repository (DOI: 10.15129/1bd18245-1226-42ed-84d9-48ae37e3d765).

\section{Acknowledgements}

The author would like to thank the volunteer computing platform IBERCIVIS for all the assistance provided during the implementation and execution of project SOLUVEL. Javier Palacios Ramos, Francisco Sanz García, Carlos Simões, Cândida Silva and Rui Brito deserve a special mention for their tireless support. IBERCIVIS was supported in part by grants from 
UMIC (Agência para a Sociedade do Conhecimento) and FCT (Fundação para a Ciência e a Tecnologia) in Portugal, and the IBERCIVIS foundation, CSIC (Consejo Superior de Investigaciones Cientificas) and Gobierno de Aragon in Spain. Special thanks are due to all the volunteers that contributed with their time and computer resources to the IBERCIVIS network.

\section{References}

[1] Westergren, J.; Lindfors, L.; Höglund, T.; Lüder, K.; Nordholm, S.; Kjellander, R.; In silico prediction of drug solubility: 1. Free energy of hydration. J. Phys. Chem. B 2007, 111, 1872-1882.

[2] Garrido, N. M.; Queimada, A. J.; Jorge, M.; Macedo, E. A.; Economou, I. G. 1Octanol/Water Partition Coefficients of n-Alkanes from Molecular Simulations of Absolute Solvation Free Energies. J. Chem. Theory Comput, 2009 5, 2436-2446.

[3] Rao, S. N.; Singh, U. C.; Bash, P. A.; Kollman, P. A. Free energy perturbation calculations on binding and catalysis after mutating Asn 155 in subtilisin. Nature 1987, 328, 551-554.

[4] Kollman, P. Free energy calculations: Applications to chemical and biochemical phenomena. Chem. Rev. 1993, 93, 2395-2417.

[5] Mobley, D. L.; Bayly, C. I.; Cooper, M. D.; Shirts, M. R.; Dill, K. A. Small molecule hydration free energies in explicit solvent: An extensive test of fixed-charge atomistic simulations. J. Chem. Theory Comput. 2009, 5, 350-358.

[6] Shivakumar, D.; Williams, J.; Wu, Y.; Damm, W.; Shelley, J.; Sherman, W. Predicition of absolute solvation free energies using molecular dynamics free energy perturbation and the OPLS force field. J. Chem. Theory Comput. 2010, 6, 1509-1519.

[7] Knight, J. L.; Yesselman, J. D.; Brooks, III, C. L. Assessing the quality of absolute hydration free energies among the CHARMM-compatible ligand parametrization schemes. $J$. Comput. Chem. 2013, 34, 893-903.

[8] Jorgensen, W. L.; Tirado-Rives, J. The OPLS potential functions for proteins. Energy minimizations for crystals of cyclic peptides and crambin. J. Am. Chem. Soc. 1988, 110, $1657-1666$.

[9] Oostenbrink, C.; Villa, A.; Mark, A. E.; van Gunsteren, W. F. A biomolecular force field based on the free enthalpy of hydration and solvation: the GROMOS force-field parameter sets 53A5 and 53A6. J. Comput. Chem. 2004, 25, 1656-1676.

[10] Schuler, L. D.; Daura, X.; van Gunsteren, W. F. An improved GROMOS96 force field for aliphatic hydrocarbons in the condensed phase. J. Comput. Chem. 2001, 22, 1205-1218.

[11] Martin, M. G.; Siepmann, J. I. Transferable potentials for phase equilibria. 1. Unitedatom description of $\mathrm{n}$-alkanes. J. Phys. Chem. B 1998, 102, 2569-2577. 
[12] Martin, M. G.; Siepmann, J. I. Novel configurational-bias Monte Carlo method for branched molecules. Transferable potentials for phase equilibria. 2. United-atom description of branched alkanes. J. Phys. Chem. B 1999, 103, 4508-4517.

[13] Lee, J.-S.; Wick, C. D.; Stubbs, J. M.; Siepmann, J. I. Simulating the vapour-liquid equilibria of large cyclic alkanes. Mol. Phys. 2005, 103, 99-104.

[14] Keasler, S. J.; Charan, S. M.; Wick, C. D.; Economou, I. G.; Siepmann, J. I. Transferable Potentials for Phase Equilibria-United Atom Description of Five- and Six-Membered Cyclic Alkanes and Ethers. J. Phys. Chem. B 2012, 116, 11234-11246.

[15] Dysthe, D. K.; Fuchs, A. H.; Rousseau, B. Fluid transport properties by equilibrium molecular dynamics. III. Evaluation of united atom interaction potential models for pure alkanes. J. Chem. Phys. 2000, 112, 7581-7590.

[16] Leontyev, I.; Stuchebrukhov, A. A. Electronic Continuum Model for Molecular Dynamics Simulations. J. Chem. Phys. 2009, 130, 085102.

[17] Jorge, M.; Garrido, N. M.; Predicting Hydrophobic Solvation by Molecular Simulation: 1. Testing United-atom Alkane Models. Submitted.

[18] Katritzky, A. R.; Oliferenko, A. A.; Oliferenko, P. V.; Petrukhin, R.; Tatham, D. B.; Maran, U.; Lomaka, A.; Acree, W. E. Jr. A General Treatment of Solubility. 1. The QSPR Correlation of Solvation Free Energies of Single Solutes in Series of Solvents. J. Chem. Inf. Comput. Sci. 2003, 43, 1794-1805.

[19] Katritzky, A. R.; Tulp, I.; Fara, D. C.; Lauria, A.; Maran, U.; Acree, W. E. Jr. A General Treatment of Solubility. 3. Principal Component Analysis (PCA) of the Solubilities of Diverse Solutes in Diverse Solvents. J. Chem. Inf. Model. 2005, 45, 913-923.

[20] Garrido, N. M.; Jorge, M., Queimada, A. J.; Macedo, E. A.; Economou, I. G. Using Molecular Simulation to Predict Solute Solvation and Partition Coefficients in Solvents of Different Polarity. Phys. Chem. Chem. Phys. 2011, 13, 9155-9164.

[21] Garrido, N. M.; Jorge, M.; Queimada, A. J.; Economou, I. G.; Macedo, E. A. Molecular Simulation of the Hydration Gibbs Energy of Barbiturates. Fluid Phase Equilibr., 2010, 289, 148-155.

[22] Garrido, N. M.; Queimada, A. J.; Jorge, M.; Economou, I. G.; Macedo, E. A. Molecular Simulation of Absolute Hydration Gibbs Energies of Polar Compounds. Fluid Phase Equilibr., 2010, 296, 110-115.

[23] Garrido, N. M.; Jorge, M.; Queimada, A. J.; Gomes, J. R. B.; Economou, I. G.; Macedo, E. A. Predicting hydration Gibbs energies of alkyl-aromatics using molecular simulation: a comparison of current force fields and the development of a new parameter set for accurate solvation data. Phys. Chem. Chem. Phys., 2011, 13, 17384-17394. 
[24] Garrido, N. M.; Queimada, A. J.; Jorge, M.; Economou, I. G.; Macedo, E. A. Prediction of the n-hexane/water and 1-octanol/water Partition Coefficients for Environmentally Relevant Compounds using Molecular Simulation. AIChE J. 2012, 58, 1929-1938.

[25] Jorge, M.; Garrido, N. M.; Queimada, A. J.; Economou, I. G.; Macedo, E. A. Effect of the Integration Method on the Accuracy and Computational Efficiency of Free Energy Calculations Using Thermodynamic Integration. J. Chem. Theory Comput. 2010, 6, 10181027.

[26] Kirkwood, J. G.; Statistical mechanics of fluid mixtures. J. Chem. Phys. 1935, 3, 300313.

[27] Hess, B.; Kutzner, C.; van der Spoel, D.; Lindahl, E. GROMACS 4: Algorithms for Highly Efficient, Load-Balanced, and Scalable Molecular Simulation. J. Chem. Theory Comput. 2008, 4, 435-447.

[28] http://www.IBERCIVIS.com/

[29] van Gunsteren, W. F.; Berendsen, H. J. C. Algorithms for Brownian Dynamics. Mol. Phys. 1982, 45, 637-647.

[30] Parrinello, M.; Rahman, A. Crystal Structure and Pair Potentials: A MolecularDynamics Study. Phys. Rev. Lett. 1980, 45, 1196-1199.

[31] van Gunsteren, W.; Berendsen, H. A leap-frog algorithm for stochastic dynamics. Mol. Simul. 1988, 1, 173-185.

[32] Paliwal, H.; Shirts, M. R. Using multistate reweighting to rapidly and efficiently explore molecular simulation parameters space for nonbonded interactions. J. Chem. Theory Comput. 2013, 9, 4700-4717.

[33] Jorgensen, W. L; Maxwell, D. S; Tirado-Rives, J. Development and Testing of the OPLS All-Atom Force Field on Conformational Energetics and Properties of Organic Liquids. $J$. Am. Chem. Soc. 1996, 118, 11225-11236.

[34] Radzicka, A.; Wolfenden, R. Comparing the polarities of the amino acids: side-chain distribution coefficients between the vapor phase, cyclohexane, 1-octanol, and neutral aqueous solution. Biochemistry 1988, 27, 1664-1670.

[35] Marenich, A. V.; Kelly, C. P.; Thompson, J. D.; Hawkins, G. D.; Chambers, C. C.; Giesen, D. J.; Winget, P.; Cramer, C. J.; Truhlar, D. G. Minnesota Solvation Database version 2012, University of Minnesota, Minneapolis, 2012.

[36] Marenich, A. V.; Olson, R. M.; Kelly, C. P.; Cramer, C. J.; Truhlar, D. G. Selfconsistent reaction field model for aqueous and nonaqueous solutions based on accurate polarized partial charges. J. Chem. Theory Comput. 2007, 3, 2011-2033.

[37] Weast, R. C.; Astle, M. J. Handbook of Data on Organic Compounds. CRC Press: Boca Raton (Fla.), USA, 1985. 
[38] NIST Chemistry webbook, http://webbook.nist.gov/chemistry/, accessed 16/10/2016.

[39] Errington, J. R.; Panagiotopoulos, A. Z. New intermolecular potential models for benzene and cyclohexane. J. Chem. Phys. 1999, 111, 9731-9738.

[40] Li, J.; Zhu, T.; Hawkins, G. D.; Winget, P.; Liotard, D. A.; Cramer, C. J.; Truhlar, D. G. Extension of the Platform of Applicability of the SM5.42R Universal Solvation Model.

Theor. Chem. Acc. 1999, 103, 9-63.

[41] Winget, P.; Hawkins, G. D.; Cramer, C. J.; Truhlar, D. G. Prediction of Vapor Pressures from Self-Solvation Free Energies Calculated by the SM5 Series of Universal Solvation Models. J. Phys. Chem. B 2000, 104, 4726-4734.

[42] Wick, C. D.; Martin, M. G.; Siepmann, J. I. Transferable potentials for phase equilibria. 4. United-atom description of linear and branched alkenes and alkylbenzenes. J. Phys. Chem. B 2000, 104, 8008 . 
Table of Contents Graphic

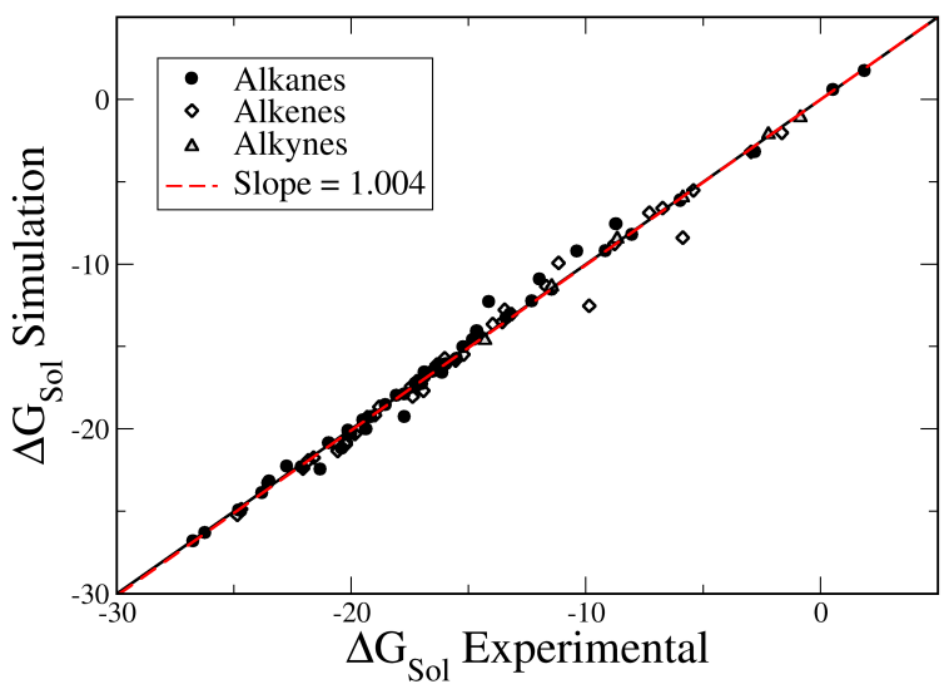

This paper presents a new united-atom model for aliphatic hydrocarbons, including alkanes, alkenes and alkynes, as well as linear, branched and cyclic molecule. Parameters were adjusted to match the solvation free energy in non-polar solvents as well as the pure liquid density, and led to good predictions of the enthalpy of vaporization of pure liquids. The new model is able to quantitatively predict the hydrophobic component of solvation free energies within experimental accuracy. 


\title{
SUPPLEMENTARY MATERIAL
}

\section{Predicting Hydrophobic Solvation by Molecular Simulation: 2. New United-atom Model for Alkanes, Alkenes and Alkynes}

\author{
Miguel Jorge* \\ Department of Chemical and Process Engineering, University of Strathclyde, 75 Montrose Street, \\ Glasgow G1 1XJ, United Kingdom \\ Email-miguel.jorge@strath.ac.uk
}

\section{S1 - Computational Methods}

For the alkane and alkene models, we have used the bonded parameters of the TraPPE force field. Unfortunately, no bonded parameters were available for alkynes in this force field. As such, we have used the bonded parameters of the OPLS-AA force field, which are provided in Table S1.

Table S1 - Bonded parameters for alkynes ${ }^{1}$, taken from the OPLS-AA force field [1]. The torsional potentials around bonds involving alkyne atoms are all zero in this model.

\begin{tabular}{l|c|c} 
Bond Stretching & $l(\mathrm{~nm})$ & $K_{1}\left(\mathrm{~kJ} \cdot \mathrm{mol}^{-1} \cdot \mathrm{nm}^{-2}\right)$ \\
\hline CZ-CZ & 0.121 & 962320 \\
CZ-CT & 0.147 & 326352 \\
\hline Angle Bending & $\theta(\mathrm{deg})$ & $K_{\theta}\left(\mathrm{kJ} \cdot \mathrm{mol}^{-1} \cdot \mathrm{rad}^{-2}\right)$ \\
\hline CZ-CZ-CT & 180 & 1255.2 \\
CZ-CT-CT & 112.7 & 488.273
\end{tabular}

${ }^{1} \mathrm{CZ}$ denotes a group with $s p$ hybridization, while $\mathrm{CT}$ denotes an $s p^{3}$ group.

Non-bonded interactions were modeled by the Lennard-Jones (LJ) potential:

$$
E_{i j}=4 \varepsilon_{i j}\left[\left(\frac{\sigma_{i j}}{r_{i j}}\right)^{12}-\left(\frac{\sigma_{i j}}{r_{i j}}\right)^{6}\right]
$$

where $r_{\mathrm{ij}}$ is the distance between two LJ interaction sites. To determine values of $\sigma_{\mathrm{ij}}$ and $\varepsilon_{\mathrm{ij}}$ for interaction between different atom types (i.e., cross interactions), we applied the Lorentz-Berthelot combination rules. For completeness, we provide all cross-interaction parameters in Tables S2 and S3. The LJ potential can also be expressed in terms of constants $C_{12}$ and $C_{6}$, which can be easily calculated from the tables of $\sigma$ and $\varepsilon$ according to the following relations:

$$
C_{12}=4 \varepsilon \sigma^{12} ; \quad C_{6}=4 \varepsilon \sigma^{6}
$$


Table S2 - Full matrix of Lennard-Jones $\sigma$ parameters for the new model (nm).

\begin{tabular}{|c|c|c|c|c|c|c|c|c|c|c|c|c|}
\hline Site & $\mathrm{CH}_{4}\left(s p^{3}\right)$ & $\mathrm{CH}_{3}\left(s p^{3}\right)$ & $\mathrm{CH}_{2}\left(s p^{3}\right)$ & $\mathrm{CH}_{2}\left(s p^{3} ; \mathrm{c}\right)$ & $\mathrm{CH}\left(s p^{3}\right)$ & $\mathrm{C}\left(s p^{3}\right)$ & $\mathrm{CH}_{2}\left(s p^{2}\right)$ & $\mathrm{CH}\left(s p^{2}\right)$ & $\mathrm{CH}\left(s p^{2} ;\right.$ conj $)$ & $\mathrm{C}\left(s p^{2}\right)$ & $\mathrm{CH}(s p)$ & $\mathrm{C}(s p)$ \\
\hline $\mathrm{CH}_{4}\left(s p^{3}\right)$ & 0.3710 & 0.3750 & 0.3850 & 0.3815 & 0.4220 & 0.5085 & 0.3693 & 0.3720 & 0.3710 & 0.3780 & 0.3513 & 0.3805 \\
\hline $\mathrm{CH}_{3}\left(s p^{3}\right)$ & 0.3750 & 0.3790 & 0.3890 & 0.3855 & 0.4260 & 0.5125 & 0.3733 & 0.3760 & 0.3750 & 0.3820 & 0.3553 & 0.3845 \\
\hline $\mathrm{CH}_{2}\left(s p^{3}\right)$ & 0.3850 & 0.3890 & 0.3990 & 0.3955 & 0.4360 & 0.5225 & 0.3833 & 0.3860 & 0.3850 & 0.3920 & 0.3653 & 0.3945 \\
\hline $\mathrm{CH}_{2}\left(s p^{3} ;\right.$ cyclic $)$ & 0.3815 & 0.3855 & 0.3955 & 0.3920 & 0.4325 & 0.5190 & 0.3798 & 0.3825 & 0.3815 & 0.3885 & 0.3618 & 0.3910 \\
\hline $\mathrm{CH}\left(s p^{3}\right)$ & 0.4220 & 0.4260 & 0.4360 & 0.4325 & 0.4730 & 0.0070 & 0.4203 & 0.4230 & 0.4220 & 0.4290 & 0.4023 & 0.4315 \\
\hline $\mathrm{C}\left(s p^{3}\right)$ & 0.5085 & 0.5125 & 0.5225 & 0.5190 & 0.5595 & 0.6460 & 0.5068 & 0.5095 & 0.5085 & 0.5155 & 0.4888 & 0.5180 \\
\hline $\mathrm{CH}_{2}\left(s p^{2}\right)$ & 0.3693 & 0.3733 & 0.3833 & 0.3798 & 0.4203 & 0.5068 & 0.3675 & 0.3703 & 0.3693 & 0.3763 & 0.3495 & 0.3788 \\
\hline $\mathrm{CH}\left(s p^{2}\right)$ & 0.3720 & 0.3760 & 0.3860 & 0.3825 & 0.4230 & 0.5095 & 0.3703 & 0.3730 & 0.3720 & 0.3790 & 0.3523 & 0.3815 \\
\hline $\begin{array}{l}\mathrm{CH}\left(s p^{2} ;\right. \\
\text { conjugated) }\end{array}$ & 0.3710 & 0.3750 & 0.3850 & 0.3815 & 0.4220 & 0.5085 & 0.3693 & 0.3720 & 0.3710 & 0.3780 & 0.3513 & 0.3805 \\
\hline $\mathrm{C}\left(s p^{2}\right)$ & 0.3780 & 0.3820 & 0.3920 & 0.3885 & 0.4290 & 0.5155 & 0.3763 & 0.3790 & 0.3780 & 0.3850 & 0.3583 & 0.3875 \\
\hline $\mathrm{CH}(s p)$ & 0.3513 & 0.3553 & 0.3653 & 0.3618 & 0.4023 & 0.4888 & 0.3495 & 0.3523 & 0.3513 & 0.3583 & 0.3315 & 0.3608 \\
\hline $\mathrm{C}(s p)$ & 0.3805 & 0.3845 & 0.3945 & 0.3910 & 0.4315 & 0.5180 & 0.3788 & 0.3815 & 0.3805 & 0.3875 & 0.3608 & 0.3900 \\
\hline
\end{tabular}


Table S3 - Full matrix of Lennard-Jones Eparameters for the new model (kJ/mol).

\begin{tabular}{|c|c|c|c|c|c|c|c|c|c|c|c|c|}
\hline Site & $\mathrm{CH}_{4}\left(s p^{3}\right)$ & $\mathrm{CH}_{3}\left(s p^{3}\right)$ & $\mathrm{CH}_{2}\left(s p^{3}\right)$ & $\mathrm{CH}_{2}\left(s p^{3} ; \mathrm{c}\right)$ & $\mathrm{CH}\left(s p^{3}\right)$ & $\mathrm{C}\left(s p^{3}\right)$ & $\mathrm{CH}_{2}\left(s p^{2}\right)$ & $\mathrm{CH}\left(s p^{2}\right)$ & $\mathrm{CH}\left(s p^{2} ;\right.$ conj $)$ & $\mathrm{C}\left(s p^{2}\right)$ & $\mathrm{CH}(s p)$ & $\mathrm{C}(s p)$ \\
\hline $\mathrm{CH}_{4}\left(s p^{3}\right)$ & 1.2000 & 0.9998 & 0.6859 & 0.7348 & 0.3194 & 0.0715 & 0.9209 & 0.6848 & 0.7203 & 0.4467 & 0.8681 & 0.6753 \\
\hline $\mathrm{CH}_{3}\left(s p^{3}\right)$ & 0.9998 & 0.8330 & 0.5714 & 0.6122 & 0.2661 & 0.0596 & 0.7673 & 0.5705 & 0.6001 & 0.3722 & 0.7233 & 0.5626 \\
\hline $\mathrm{CH}_{2}\left(s p^{3}\right)$ & 0.6859 & 0.5714 & 0.3920 & 0.4200 & 0.1825 & 0.0409 & 0.5263 & 0.3914 & 0.4117 & 0.2553 & 0.4962 & 0.3860 \\
\hline $\mathrm{CH}_{2}\left(s p^{3} ;\right.$ cyclic $)$ & 0.7348 & 0.6122 & 0.4200 & 0.4500 & 0.1956 & 0.0438 & 0.5639 & 0.4193 & 0.4411 & 0.2735 & 0.5316 & 0.4135 \\
\hline $\mathrm{CH}\left(s p^{3}\right)$ & 0.3194 & 0.2661 & 0.1825 & 0.1956 & 0.0850 & 0.0070 & 0.2451 & 0.1822 & 0.1917 & 0.1189 & 0.2310 & 0.1797 \\
\hline $\mathrm{C}\left(s p^{3}\right)$ & 0.0715 & 0.0596 & 0.0409 & 0.0438 & 0.0190 & 0.0043 & 0.0549 & 0.0408 & 0.0429 & 0.0266 & 0.0517 & 0.0402 \\
\hline $\mathrm{CH}_{2}\left(s p^{2}\right)$ & 0.9209 & 0.7673 & 0.5263 & 0.5639 & 0.2451 & 0.0549 & 0.7067 & 0.5255 & 0.5527 & 0.3428 & 0.6662 & 0.5182 \\
\hline $\mathrm{CH}\left(s p^{2}\right)$ & 0.6848 & 0.5705 & 0.3914 & 0.4193 & 0.1822 & 0.0408 & 0.5255 & 0.3908 & 0.4110 & 0.2549 & 0.4954 & 0.3853 \\
\hline $\begin{array}{l}\mathrm{CH}\left(s p^{2} ;\right. \\
\text { conjugated) }\end{array}$ & 0.7203 & 0.6001 & 0.4117 & 0.4411 & 0.1917 & 0.0429 & 0.5527 & 0.4110 & 0.4323 & 0.2681 & 0.5211 & 0.4053 \\
\hline $\mathrm{C}\left(s p^{2}\right)$ & 0.4467 & 0.3722 & 0.2553 & 0.2735 & 0.1189 & 0.0266 & 0.3428 & 0.2549 & 0.2681 & 0.1663 & 0.3231 & 0.2514 \\
\hline $\mathrm{CH}(s p)$ & 0.8681 & 0.7233 & 0.4962 & 0.5316 & 0.2310 & 0.0517 & 0.6662 & 0.4954 & 0.5211 & 0.3231 & 0.6280 & 0.4885 \\
\hline $\mathrm{C}(s p)$ & 0.6753 & 0.5626 & 0.3860 & 0.4135 & 0.1797 & 0.0402 & 0.5182 & 0.3853 & 0.4053 & 0.2514 & 0.4885 & 0.3800 \\
\hline
\end{tabular}




\section{S2 - Force-field Validation}

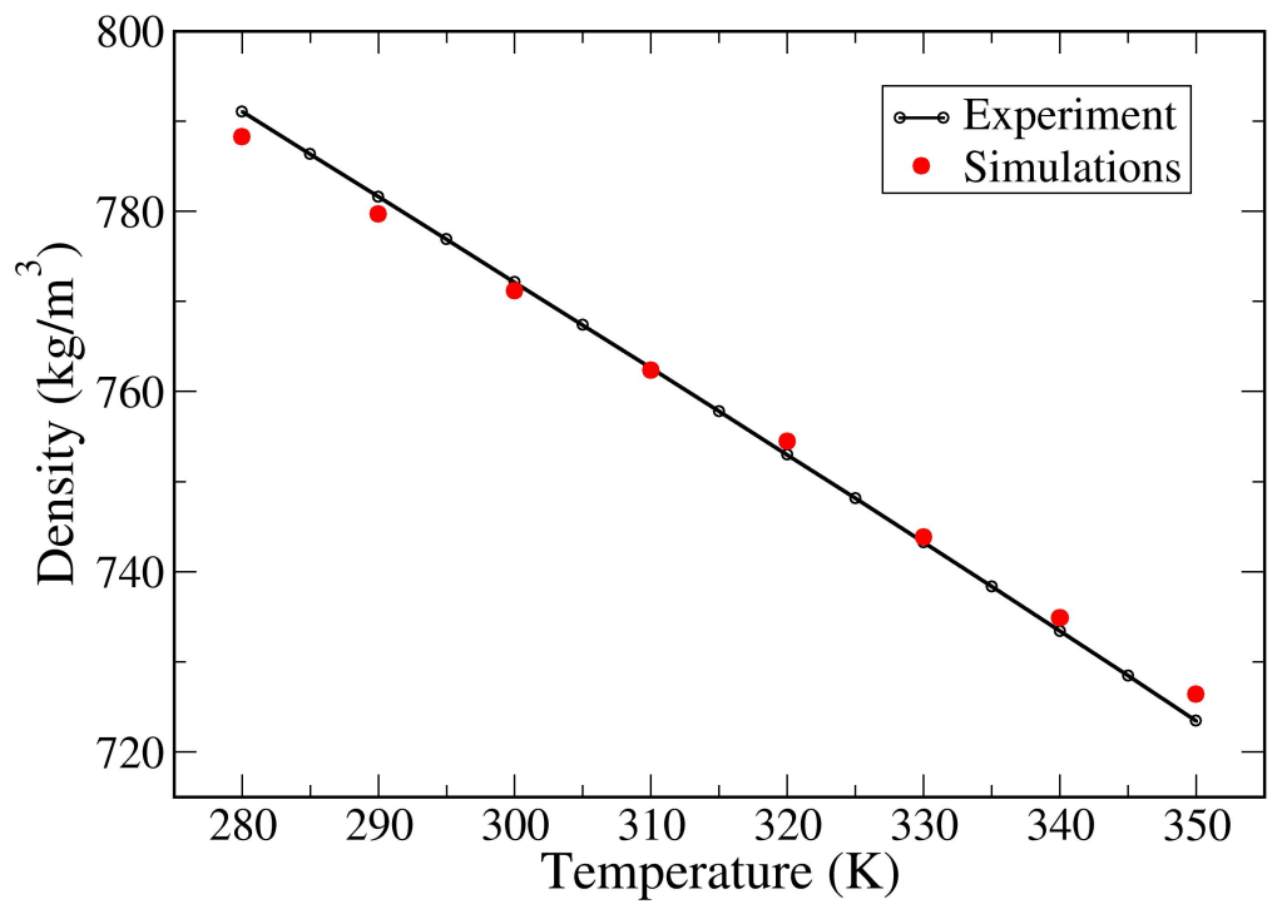

Figure S1 - Density of pure cyclohexane as a function of temperature from experiment (black line) and simulations using the new model developed in this work (red circles).

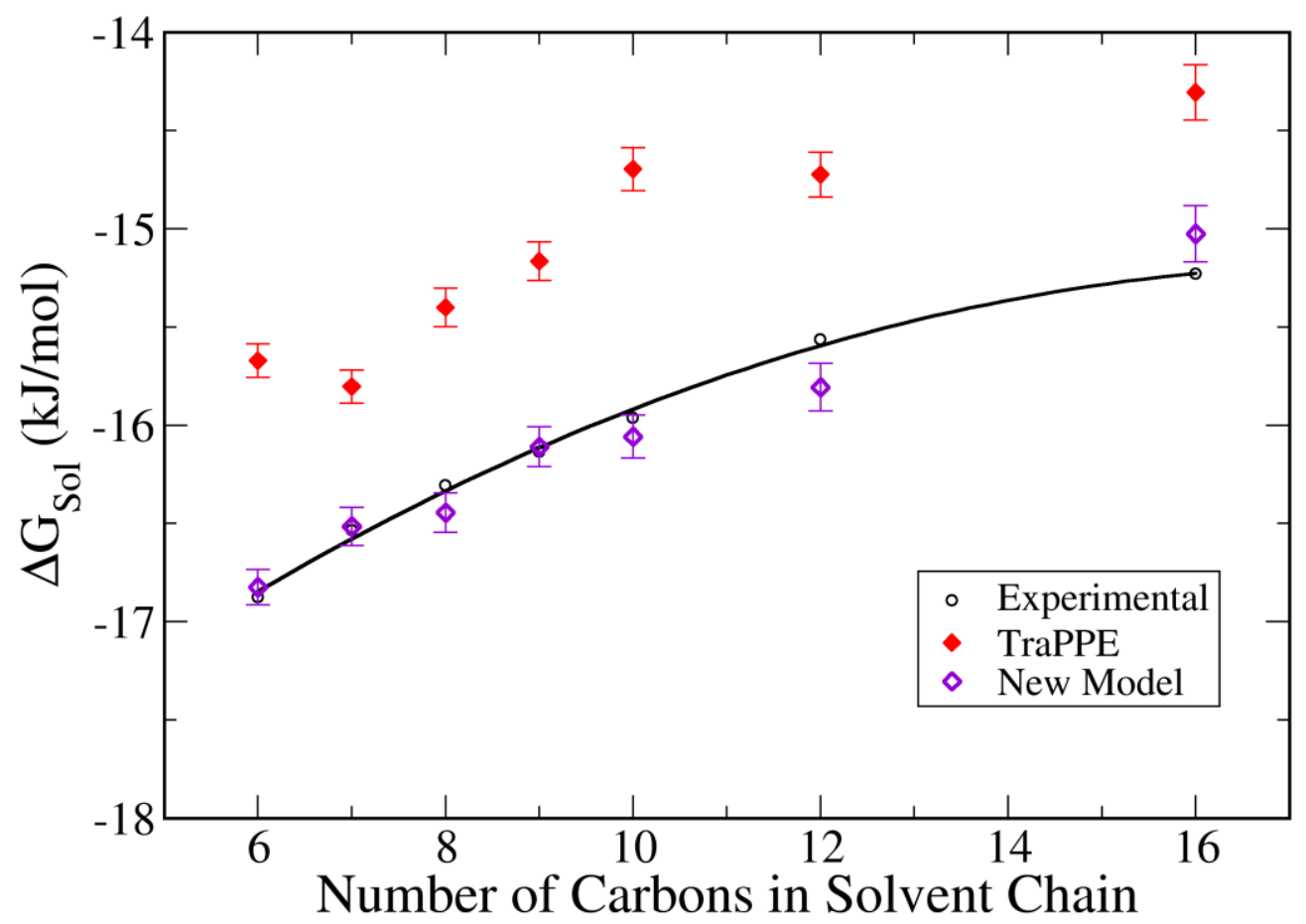

Figure S2 - Comparison between the original TraPPE model and the new model for solvation of $n$ hexane solute in linear alkane solvents of different chain length. 


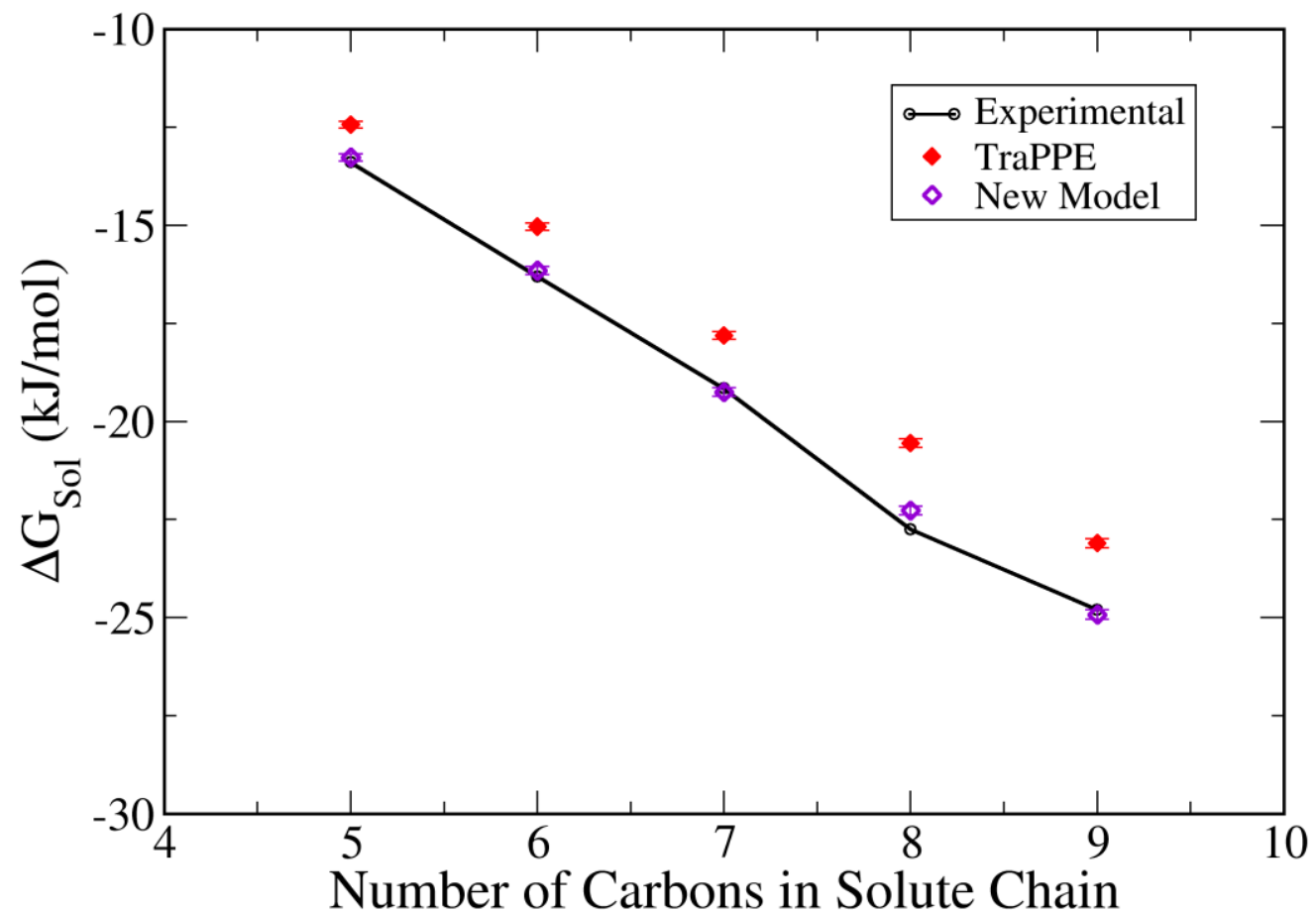

Figure S3 - Comparison between the original TraPPE model and the new model for linear alkane solutes of different chain length in 2,2,4-trimethylpentane solvent.

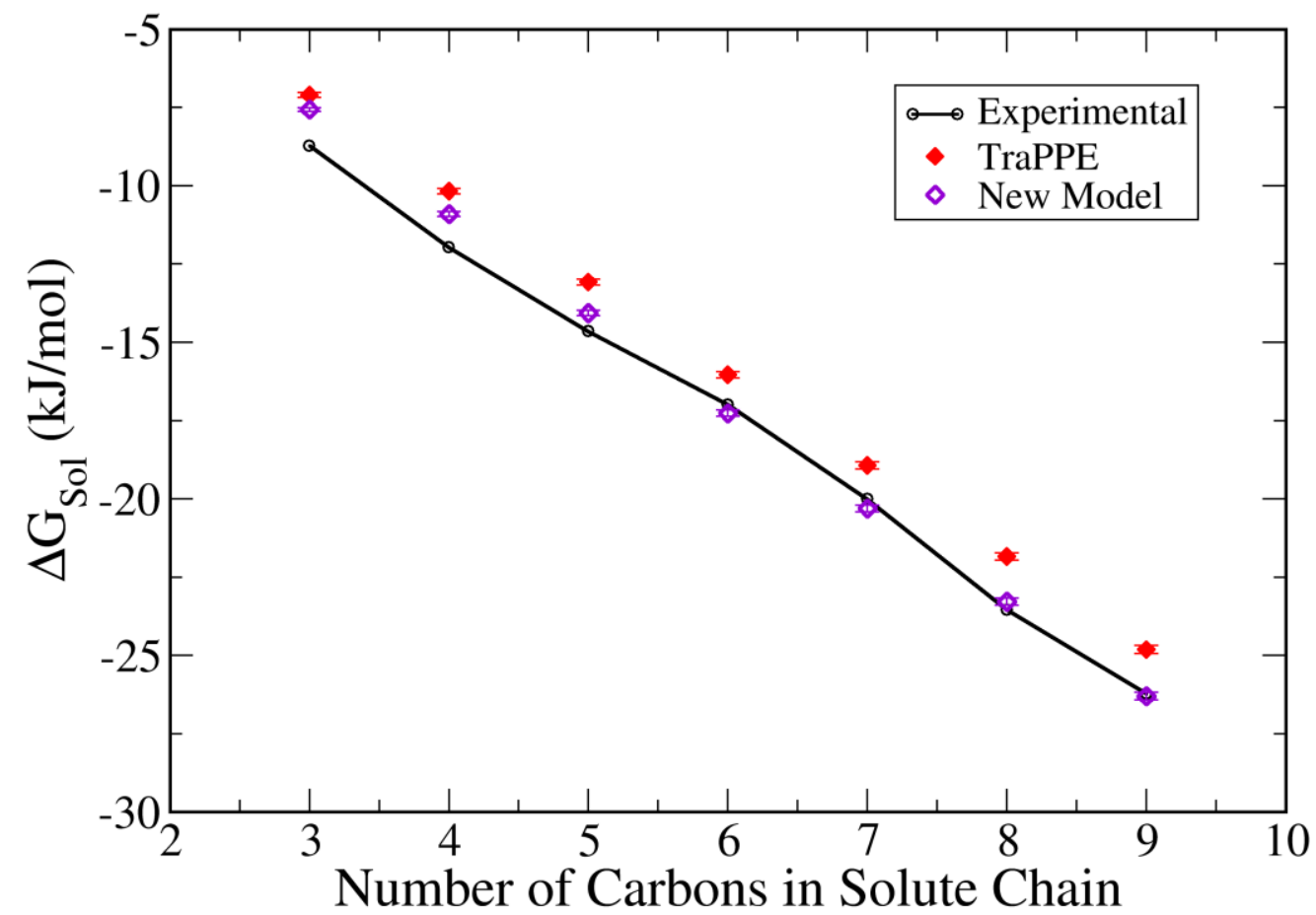

Figure S4 - Comparison between the original TraPPE model and the new model for linear alkane solutes of different chain length in cyclohexane solvent. 


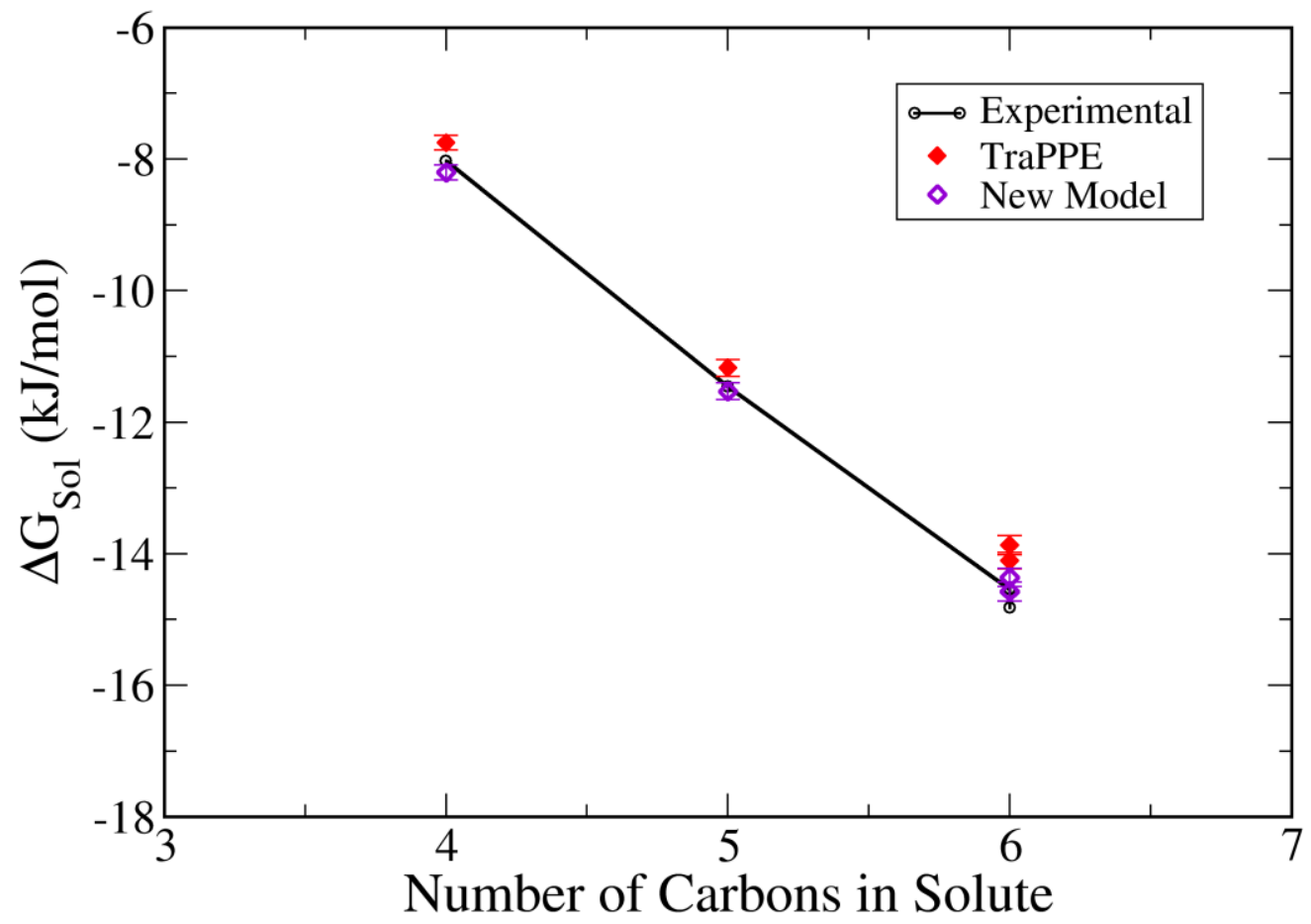

Figure S5 - Comparison between the original TraPPE model and the new model for single-branched alkane solutes in n-hexadecane solvent. For hexane isomers in experiments and simulations, the point with the lowest free energy corresponds to 3-methylpentane, while the point with the highest free energy corresponds to 2-methylpentane.

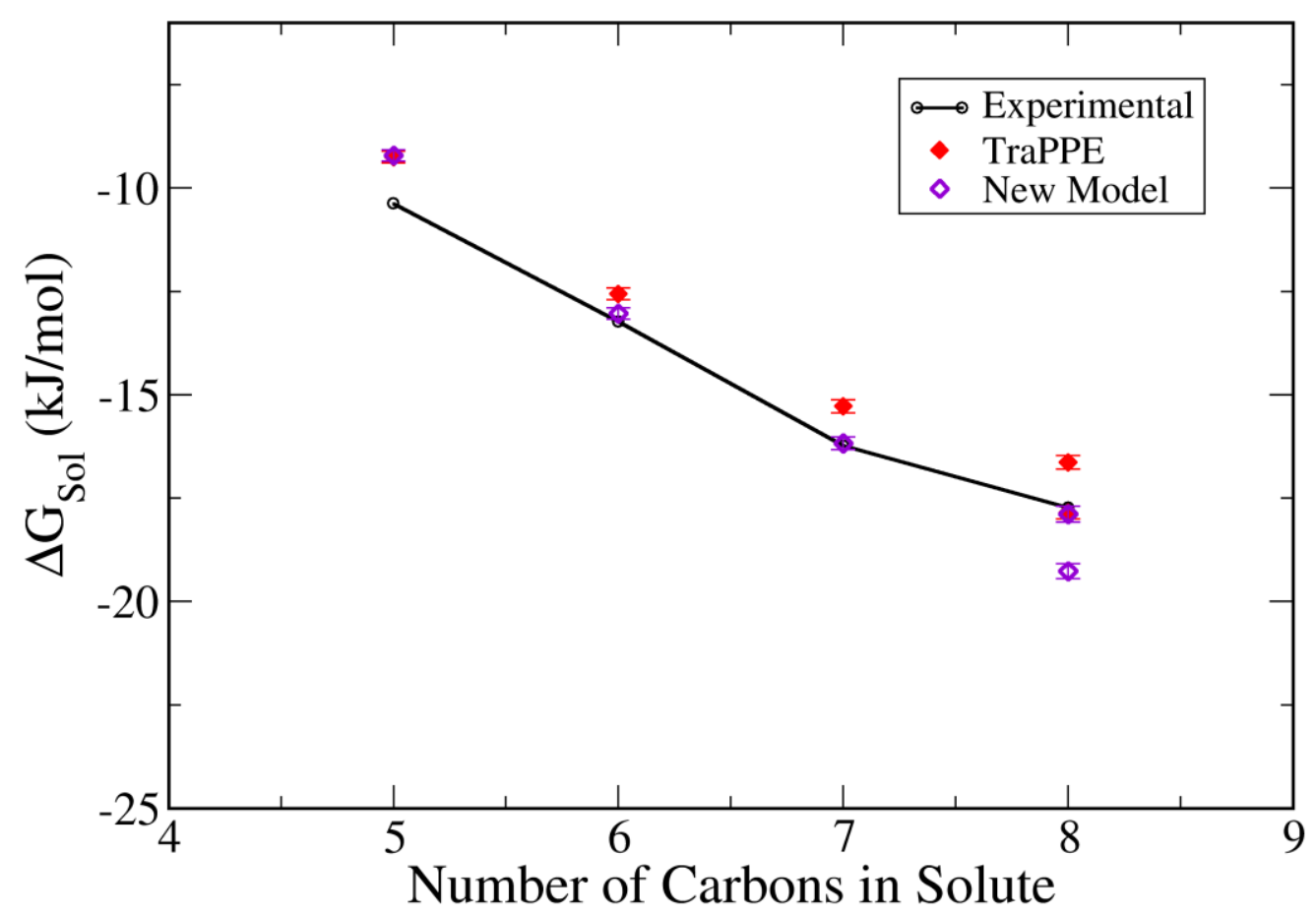

Figure S6 - Comparison between the original TraPPE model and the modified version for doublebranched alkane solutes in n-hexadecane solvent. For octane isomers in experiments and all calculations, the point with the lowest free energy corresponds to 2,2,3-trimethylpentane, while the point with the highest free energy corresponds to 2,2,4-trimethylpentane. 


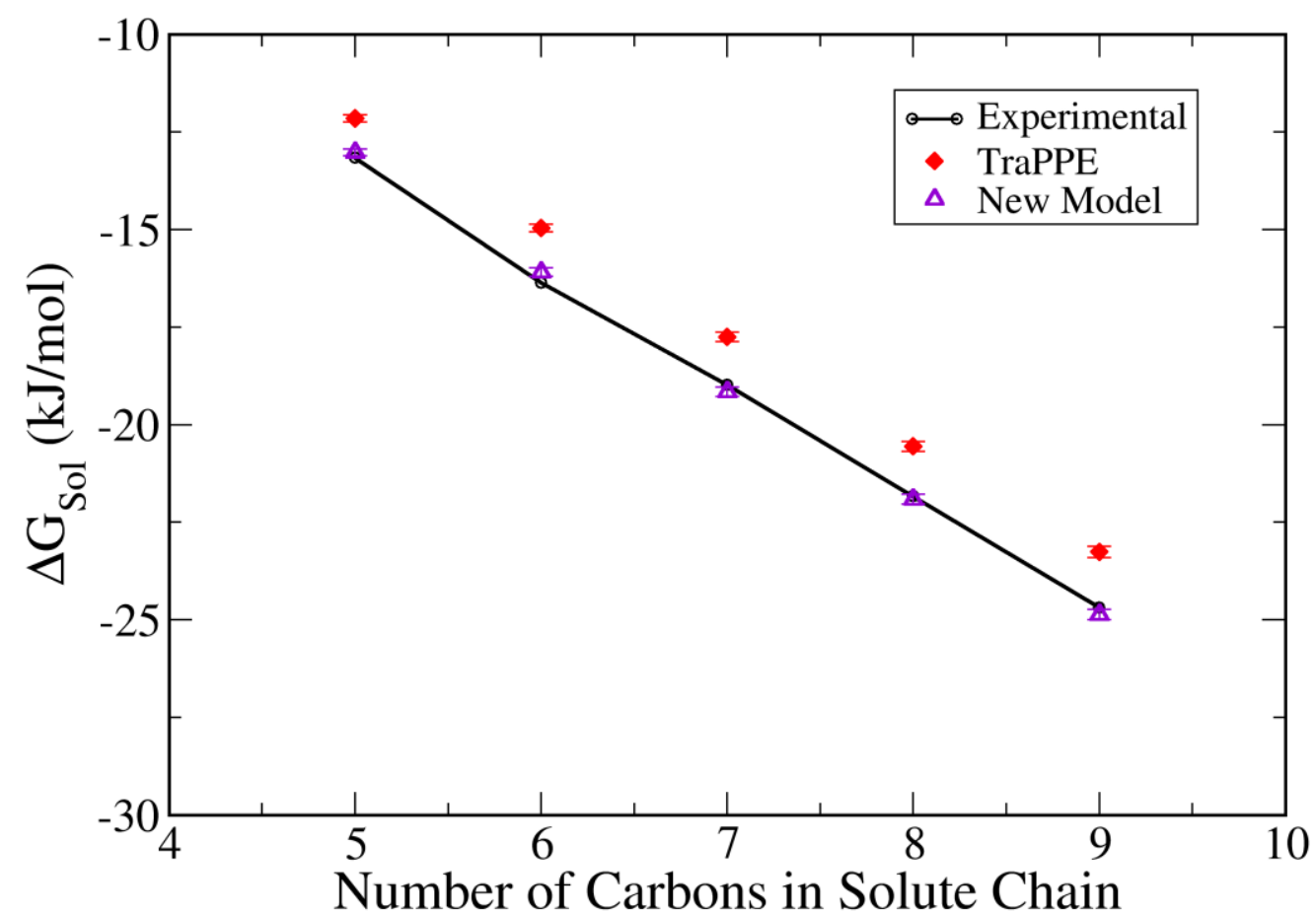

Figure S7 - Comparison between the original TraPPE model and the new model for linear alkane solutes of different chain length in 1-decene solvent.

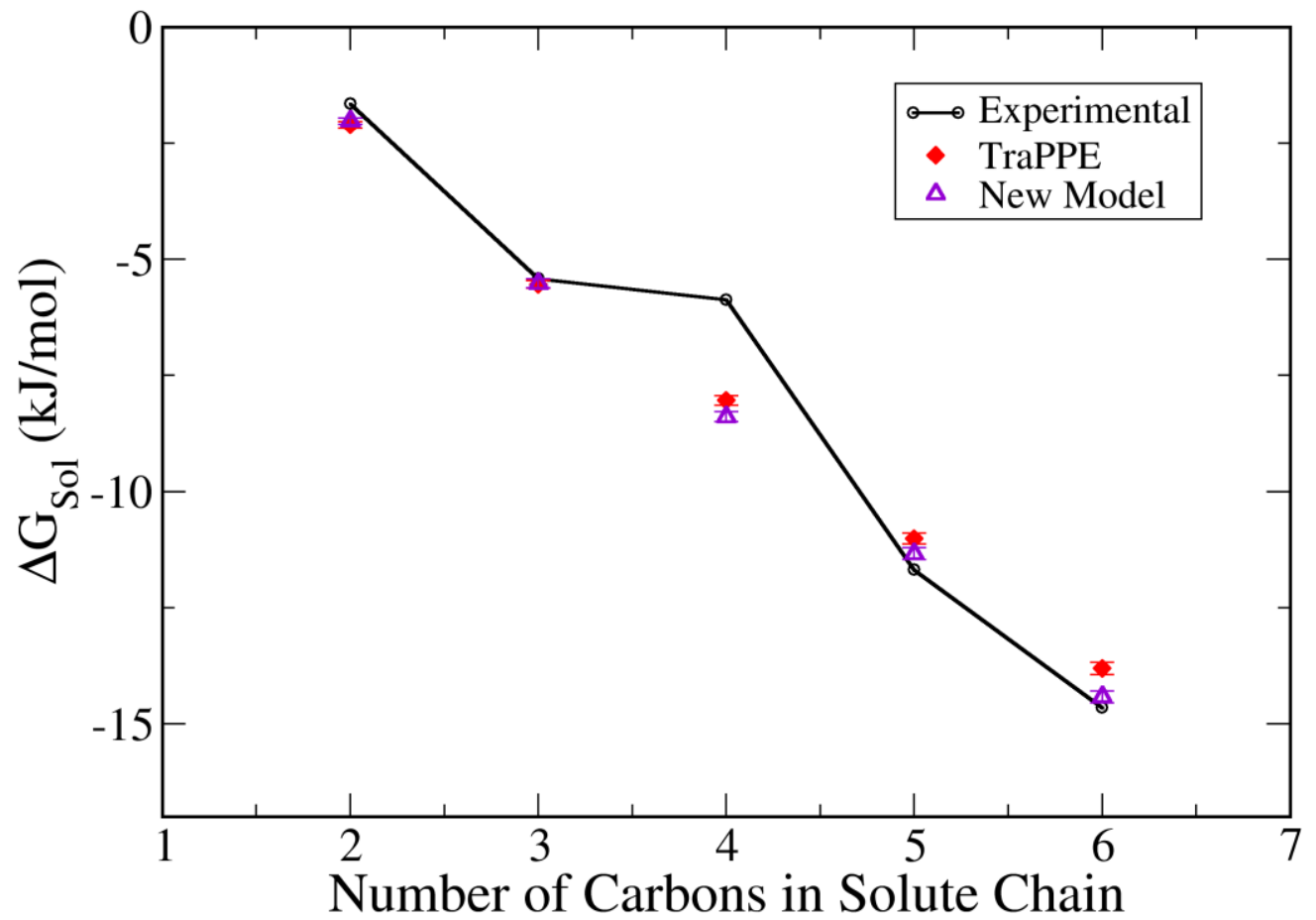

Figure S8 - Comparison between the original TraPPE model and the new model for linear alkene solutes of different chain length in n-hexadecane solvent. 


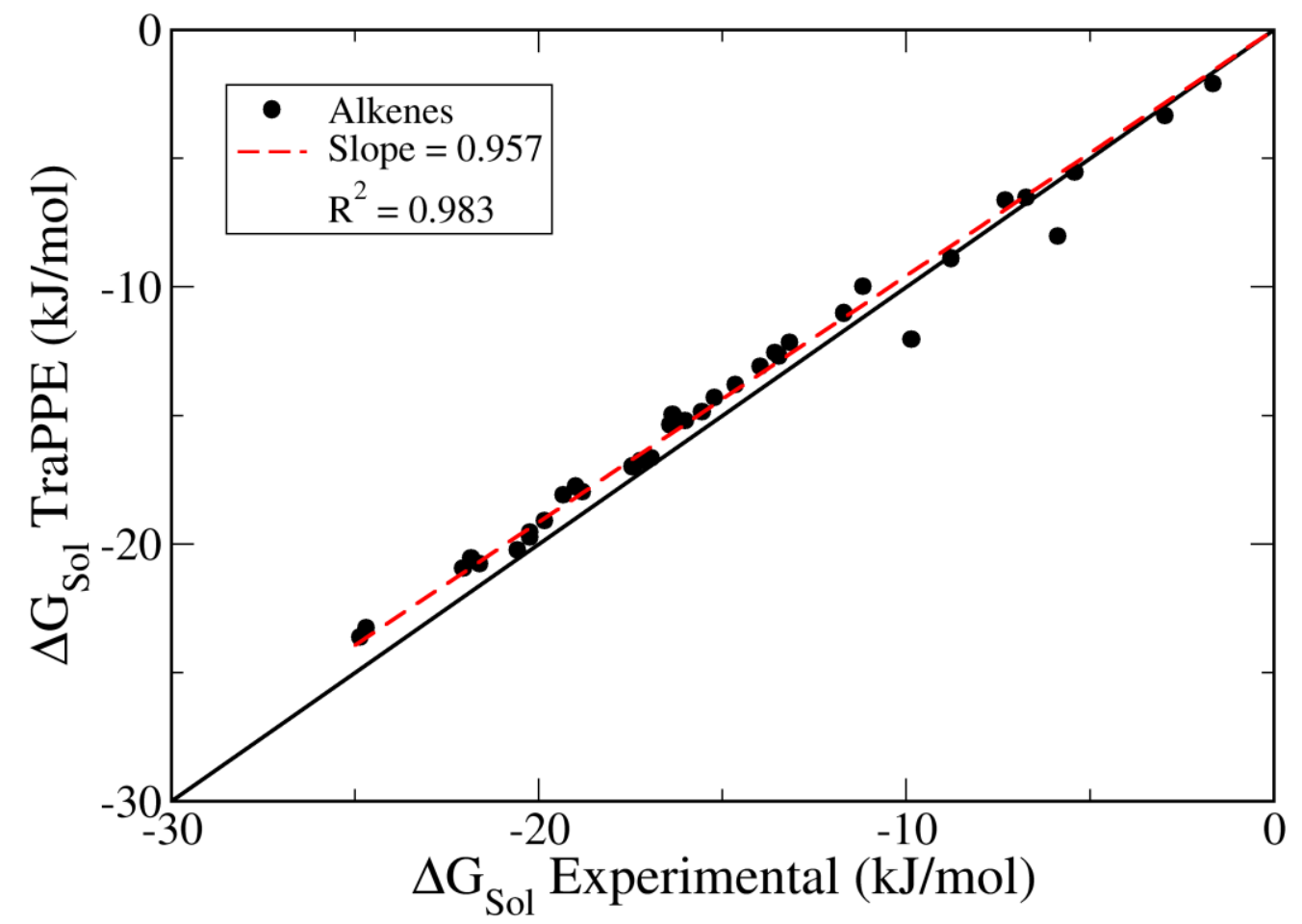

Figure S9 - Comparison between experimental and simulated solvation free energies for the entire alkene data set using the TraPPE force-field. The dashed red line shows a linear fit (with forced intercept at the origin) through the data. We report also the slope and the correlation coefficient of the fit. 
Table S4 - Comparison between experimental solvation free energies and those calculated using the new model for the entire data set of alkanes, alkenes and alkynes examined in this paper. All values are in $\mathrm{kJ} / \mathrm{mol}$. Experimental data are from refs [1] and [2], except where noted. Uncertainty in the simulated free energies is reported as \pm the standard error. ASD = absolute signed deviation between simulation and experiment. The first section includes pairs involving only alkanes, the second section includes pairs that involve at least one alkene, and the third section includes pairs that involve at least one alkyne.

\begin{tabular}{|c|c|c|c|c|}
\hline Solute & Solvent & $\Delta \mathrm{G}_{\exp }$ & $\Delta \mathrm{G}_{\text {sim }}$ & ASD \\
\hline methane & hexadecane & 1.88 & $1.744 \pm 0.064$ & 0.139 \\
\hline ethane & hexadecane & -2.80 & $-3.175 \pm 0.076$ & 0.372 \\
\hline propane & hexadecane & -5.98 & $-6.117 \pm 0.094$ & 0.134 \\
\hline butane & hexadecane & -9.16 & $-9.184 \pm 0.113$ & 0.021 \\
\hline pentane & hexadecane & -12.30 & $-12.230 \pm 0.125$ & -0.071 \\
\hline hexane & hexadecane & -15.23 & $-15.026 \pm 0.143$ & -0.204 \\
\hline heptane & hexadecane & -18.07 & $-17.969 \pm 0.148$ & -0.106 \\
\hline octane & hexadecane & -20.96 & $-20.849 \pm 0.167$ & -0.113 \\
\hline nonane & hexadecane & -23.81 & $-23.883 \pm 0.163$ & 0.076 \\
\hline decane & hexadecane & -26.74 & $-26.803 \pm 0.585$ & 0.067 \\
\hline hexane & hexane & -16.88 & $-16.824 \pm 0.090$ & -0.052 \\
\hline hexane & heptane & -16.53 & $-16.515 \pm 0.096$ & -0.019 \\
\hline hexane & octane & -16.31 & $-16.444 \pm 0.100$ & 0.138 \\
\hline hexane & nonane & -16.13 & $-16.110 \pm 0.102$ & -0.025 \\
\hline hexane & decane & -15.96 & $-16.058 \pm 0.109$ & 0.094 \\
\hline hexane & dodecane & -15.56 & $-15.807 \pm 0.121$ & 0.242 \\
\hline heptane & heptane & -19.50 & $-19.462 \pm 0.097$ & -0.036 \\
\hline octane & octane & -22.12 & $-22.310 \pm 0.122$ & 0.189 \\
\hline nonane & nonane & -24.69 & $-24.960 \pm 0.136$ & 0.273 \\
\hline isobutane & hexadecane & -8.03 & $-8.203 \pm 0.064$ & 0.170 \\
\hline isopentane & hexadecane & -11.46 & $-11.530 \pm 0.076$ & 0.066 \\
\hline neopentane & hexadecane & -10.38 & $-9.214 \pm 0.094$ & -1.162 \\
\hline 2-methylpentane & hexadecane & -14.54 & $-14.361 \pm 0.116$ & -0.177 \\
\hline 3-methylpentane & hexadecane & -14.82 & $-14.574 \pm 0.125$ & -0.249 \\
\hline 2,2-dimethylbutane & hexadecane & -13.23 & $-13.036 \pm 0.133$ & -0.191 \\
\hline 2,3-dimethylpentane & hexadecane & -17.22 & $-17.386 \pm 0.139$ & 0.168 \\
\hline 2,2,3-trimethylbutane & hexadecane & -16.23 & $-16.174 \pm 0.145$ & -0.060 \\
\hline 2,3,4-trimethylpentane & hexadecane & -19.38 & $-20.006 \pm 0.139$ & 0.622 \\
\hline 2,2,4-trimethylpentane & hexadecane & -17.73 & $-17.885 \pm 0.153$ & 0.154 \\
\hline 2,2,3-trimethylpentane & hexadecane & -17.74 & $-19.263 \pm 0.157$ & 1.523 \\
\hline pentane & 2,2,4-trimethylpentane & -13.40 & $-13.272 \pm 0.178$ & -0.126 \\
\hline hexane & 2,2,4-trimethylpentane & -16.31 & $-16.156 \pm 0.192$ & -0.150 \\
\hline
\end{tabular}




\begin{tabular}{|c|c|c|c|c|}
\hline Solute & Solvent & $\Delta \mathrm{G}_{\exp }$ & $\Delta \mathrm{G}_{\mathrm{sim}}$ & ASD \\
\hline heptane & 2,2,4-trimethylpentane & -19.16 & $-19.253 \pm 0.180$ & 0.097 \\
\hline octane & 2,2,4-trimethylpentane & -22.75 & $-22.268 \pm 0.092$ & -0.480 \\
\hline nonane & 2,2,4-trimethylpentane & -24.80 & $-24.922 \pm 0.104$ & 0.121 \\
\hline 2-methylpentane & 2,2,4-trimethylpentane & -15.51 & $-15.721 \pm 0.108$ & 0.214 \\
\hline 2,3,4-trimethylpentane & 2,2,4-trimethylpentane & -20.41 & $-21.167 \pm 0.107$ & 0.756 \\
\hline cyclopentane & hexadecane & -14.14 & $-12.280 \pm 0.122$ & -1.859 \\
\hline cyclohexane & hexadecane & -16.88 & $-16.554 \pm 0.130$ & -0.322 \\
\hline cycloheptane & hexadecane & -20.13 & $-20.093 \pm 0.161$ & -0.033 \\
\hline cyclooctane & hexadecane & -23.49 & $-23.170 \pm 0.145$ & -0.319 \\
\hline cyclohexane & 2,2,4-trimethylpentane & -17.27 & $-17.248 \pm 0.097$ & -0.027 \\
\hline methane & cyclohexane & $0.54^{*}$ & $0.584 \pm 0.049$ & -0.044 \\
\hline propane & cyclohexane & -8.72 & $-7.561 \pm 0.056$ & -1.162 \\
\hline butane & cyclohexane & -11.97 & $-10.903 \pm 0.085$ & -1.070 \\
\hline pentane & cyclohexane & -14.65 & $-14.064 \pm 0.092$ & -0.588 \\
\hline hexane & cyclohexane & -16.99 & $-17.265 \pm 0.105$ & 0.275 \\
\hline heptane & cyclohexane & -20.01 & $-20.307 \pm 0.109$ & 0.295 \\
\hline octane & cyclohexane & -23.55 & $-23.285 \pm 0.120$ & -0.261 \\
\hline nonane & cyclohexane & -26.23 & $-26.303 \pm 0.124$ & 0.077 \\
\hline 2-methylpentane & cyclohexane & -16.13 & $-16.568 \pm 0.111$ & 0.433 \\
\hline 2,3,4-trimethylpentane & cyclohexane & -21.32 & $-22.440 \pm 0.124$ & 1.117 \\
\hline cyclohexane & cyclohexane & -18.54 & $-18.529 \pm 0.110$ & -0.006 \\
\hline pentane & 1-octene & -13.57 & $-13.505 \pm 0.093$ & -0.064 \\
\hline n-hexane & 1-octene & -16.42 & $-16.395 \pm 0.101$ & -0.025 \\
\hline n-heptane & 1-octene & -19.33 & $-19.284 \pm 0.107$ & -0.043 \\
\hline n-octane & 1-octene & -22.06 & $-22.399 \pm 0.113$ & 0.335 \\
\hline n-nonane & 1-octene & -24.86 & $-25.210 \pm 0.121$ & 0.35 \\
\hline 2-methylpentane & 1-octene & -15.56 & $-15.839 \pm 0.103$ & 0.27 \\
\hline 2,4-dimethylpentane & 1-octene & -17.39 & $-18.028 \pm 0.112$ & 0.64 \\
\hline 2,5-dimethylhexane & 1-octene & -20.24 & $-20.801 \pm 0.118$ & 0.56 \\
\hline 2,3,4-trimethylpentane & 1-octene & -20.58 & $-21.327 \pm 0.109$ & 0.75 \\
\hline cyclohexane & 1-octene & -17.45 & $-17.415 \pm 0.088$ & -0.031 \\
\hline pentane & 1-decene & -13.17 & $-13.024 \pm 0.086$ & -0.146 \\
\hline n-hexane & 1-decene & -16.36 & $-16.091 \pm 0.111$ & -0.272 \\
\hline n-heptane & 1-decene & -18.99 & $-19.160 \pm 0.118$ & 0.175 \\
\hline n-octane & 1-decene & -21.84 & $-21.910 \pm 0.124$ & 0.074 \\
\hline n-nonane & 1-decene & -24.69 & $-24.863 \pm 0.134$ & 0.176 \\
\hline 2-methylpentane & 1-decene & -15.22 & $-15.477 \pm 0.106$ & 0.255 \\
\hline 2,4-dimethylpentane & 1-decene & -16.93 & $-17.680 \pm 0.125$ & 0.747 \\
\hline 2,5-dimethylhexane & 1-decene & -19.84 & $-20.301 \pm 0.130$ & 0.461 \\
\hline 2,3,4-trimethylpentane & 1-decene & -20.24 & $-20.945 \pm 0.130$ & 0.705 \\
\hline
\end{tabular}




\begin{tabular}{l|l|r|r|r}
\multicolumn{1}{c|}{ Solute } & \multicolumn{1}{|c|}{ Solvent } & \multicolumn{1}{c|}{$\Delta \mathrm{G}_{\exp }$} & \multicolumn{1}{c|}{$\Delta \mathrm{G}_{\text {sim }}$} & \multicolumn{1}{c}{ ASD } \\
\hline cyclohexane & 1-decene & -17.22 & $-17.093 \pm 0.098$ & -0.125 \\
ethene & n-heptane & -2.96 & $-3.179 \pm 0.055$ & 0.214 \\
propylene & n-heptane & -7.30 & $-6.873 \pm 0.064$ & -0.425 \\
1-hexene & n-heptane & -16.02 & $-15.711 \pm 0.086$ & -0.310 \\
1-heptene & n-heptane & -18.81 & $-18.653 \pm 0.097$ & -0.161 \\
1-octene & n-heptane & -21.61 & $-21.744 \pm 0.106$ & 0.136 \\
1,3-butadiene & n-heptane & -11.17 & $-9.926 \pm 0.070$ & -1.249 \\
2-methyl-2-butene & n-heptane & -13.97 & $-13.634 \pm 0.085$ & -0.334 \\
isoprene & n-heptane & -13.46 & $-12.772 \pm 0.079$ & -0.683 \\
propylene & 2,2,4-trimethylpentane & -6.73 & $-6.597 \pm 0.066$ & -0.131 \\
1-pentene & 2,2,4-trimethylpentane & -9.86 & $-12.521 \pm 0.093$ & 2.658 \\
ethene & hexadecane & -1.65 & $-2.025 \pm 0.069$ & 0.372 \\
propylene & hexadecane & -5.42 & $-5.519 \pm 0.096$ & 0.103 \\
1-butene & hexadecane & -5.87 & $-8.388 \pm 0.105$ & 2.516 \\
1-pentene & hexadecane & -11.69 & $-11.337 \pm 0.127$ & -0.351 \\
1-hexene & hexadecane & -14.65 & $-14.418 \pm 0.128$ & -0.234 \\
1,3-butadiene & hexadecane & -8.78 & $-8.763 \pm 0.099$ & -0.017 \\
\hline acetylene & hexadecane & -0.86 & $-1.102 \pm 0.060$ & 0.247 \\
propyne & hexadecane & -5.87 & $-5.786 \pm 0.097$ & -0.086 \\
1-butyne & hexadecane & -8.67 & $-8.733 \pm 0.106$ & 0.067 \\
1-pentyne & hexadecane & -11.46 & $-11.460 \pm 0.129$ & 0.000 \\
1-hexyne & hexadecane & -14.31 & $-14.639 \pm 0.143$ & 0.329 \\
acetylene & n-heptane & -2.22 & $-2.138 \pm 0.046$ & -0.086 \\
* Taken from ref [3] & & & \\
& & & & \\
& & & & \\
& & & &
\end{tabular}


Table S5 - Comparison between experimental densities [4] and those calculated using the new model for all alkanes, alkenes and alkynes examined in this paper. All values are in $\mathrm{kg} / \mathrm{m}^{3}$. Uncertainty in the simulated densities is reported as \pm the standard error.

\begin{tabular}{l|r|r}
\multicolumn{1}{c|}{ Solvent } & \multicolumn{1}{c|}{ Exp } & \multicolumn{1}{c}{ Simulation } \\
\hline hexane & 654.9 & $654.9 \pm 0.32$ \\
heptane & 679.7 & $680.8 \pm 0.16$ \\
octane & 698.4 & $700.6 \pm 0.16$ \\
nonane & 714.2 & $716.9 \pm 0.24$ \\
decane & 726.6 & $729.2 \pm 0.14$ \\
dodecane & 745.8 & $749.2 \pm 0.39$ \\
hexadecane & 770.3 & $776.0 \pm 0.24$ \\
2,2,4-trimethylpentane & 687.8 & $690.9 \pm 0.21$ \\
cyclohexane & 774.0 & $772.5 \pm 0.20$ \\
ethane & 568.0 & $567.4 \pm 0.23$ \\
propene & 609.4 & $604.8 \pm 0.61$ \\
1-butene & 625.6 & $621.0 \pm 0.18$ \\
1-hexene & 671.0 & $668.9 \pm 0.36$ \\
1-octene & 710.4 & $714.2 \pm 0.31$ \\
1,3-butadiene & 615.2 & $617.1 \pm 0.33$ \\
acetylene & 620.8 & $619.9 \pm 0.19$ \\
propyne & 706.2 & $703.7 \pm 1.0$ \\
1-butyne & 678.4 & $723.8 \pm 2.5$ \\
1-pentyne & 690.1 & $688.3 \pm 1.7$ \\
1-hexyne & 715.5 & $716.7 \pm 1.3$
\end{tabular}

\section{References}

[1] Katritzky, A. R.; Oliferenko, A. A.; Oliferenko, P. V.; Petrukhin, R.; Tatham, D. B.; Maran, U.; Lomaka, A.; Acree, W. E. Jr. A General Treatment of Solubility. 1. The QSPR Correlation of Solvation Free Energies of Single Solutes in Series of Solvents. J. Chem. Inf. Comput. Sci. 2003, 43, 1794-1805.

[2] Katritzky, A. R.; Tulp, I.; Fara, D. C.; Lauria, A.; Maran, U.; Acree, W. E. Jr. A General Treatment of Solubility. 3. Principal Component Analysis (PCA) of the Solubilities of Diverse Solutes in Diverse Solvents. J. Chem. Inf. Model. 2005, 45, 913-923.

[3] Radzicka, A.; Wolfenden, R. Comparing the polarities of the amino acids: side-chain distribution coefficients between the vapor phase, cyclohexane, 1-octanol, and neutral aqueous solution. Biochemistry 1988, 27, 1664-1670.

[4] Weast, R. C.; Astle, M. J. Handbook of Data on Organic Compounds. CRC Press: Boca Raton (Fla.), USA, 1985. 
